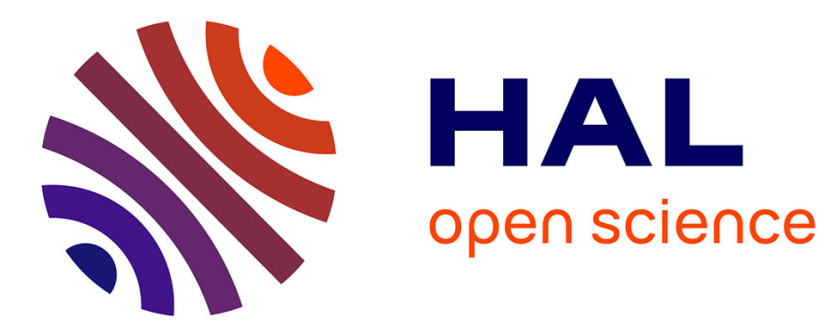

\title{
Quasipatterns in a parametrically forced horizontal fluid film
}

\author{
Médéric Argentina, Gérard Iooss
}

\section{To cite this version:}

Médéric Argentina, Gérard Iooss. Quasipatterns in a parametrically forced horizontal fluid film. Physica D: Nonlinear Phenomena, 2012, 10.1016/j.physd.2012.04.011 . hal-01265170

\section{HAL Id: hal-01265170 \\ https://hal.univ-cotedazur.fr/hal-01265170}

Submitted on 31 Jan 2016

HAL is a multi-disciplinary open access archive for the deposit and dissemination of scientific research documents, whether they are published or not. The documents may come from teaching and research institutions in France or abroad, or from public or private research centers.
L'archive ouverte pluridisciplinaire HAL, est destinée au dépôt et à la diffusion de documents scientifiques de niveau recherche, publiés ou non, émanant des établissements d'enseignement et de recherche français ou étrangers, des laboratoires publics ou privés. 


\title{
Quasipatterns in a parametrically forced horizontal fluid film
}

\author{
Médéric Argentina, Gérard Iooss* \\ LJAD, UMR 6621 CNRS UNS, Université de Nice - Sophia Antipolis, \\ 06108 Nice Cedex 02, France
}

\begin{abstract}
We shake harmonically a thin horizontal viscous fluid layer (frequency forcing $\Omega$, only one harmonic), to reproduce the Faraday experiment and using the system derived in ${ }^{31}$ invariant under horizontal rotations. When the physical parameters are suitably chosen, there is a critical value of the amplitude of the forcing such that instability occurs with at the same time the mode oscillating at frequency $\Omega / 2$, and the mode with frequency $\Omega$. Moreover, at criticality the corresponding wave lengths $k_{c}$ and $k_{c}^{\prime}$ are such that if we define the family of $2 q$ equally spaced (horizontal) wave vectors $\mathbf{k}_{j}$ on the circle of radius $k_{c}$, then $\mathbf{k}_{j}+\mathbf{k}_{l}=\mathbf{k}_{n}^{\prime}$, with $\left|\mathbf{k}_{j}\right|=\left|\mathbf{k}_{l}\right|=k_{c}, \quad\left|\mathbf{k}_{n}^{\prime}\right|=k_{c}^{\prime}$.

It results under the above conditions that 0 is an eigenvalue of the linearized operator in a space of time-periodic functions (frequency $\Omega / 2$ ) having a spatially quasiperiodic pattern if $q \geq 4$. Restricting our study to solutions invariant under rotations of angle $2 \pi / q$, gives a kernel of dimension 4 .

In the spirit of ${ }^{26}$ we derive formally amplitude equations for perturbations possessing this symmetry. Then we give simple necessary conditions on coefficients, for obtaining the bifurcation of (formally) stable time-periodic (frequency $\Omega / 2$ ) quasipatterns. In particular, we obtain a solution such that a time shift by half the period, is equivalent to a rotation of angle $\pi / q$ of the pattern.
\end{abstract}

We finally explain why this derivation is formal, no center manifold reduction being available, because of the occurence of small divisors ( $\sec ^{12}$ ), and the fact that the 0 Floquet exponent is not isolated in the spectrum of

\footnotetext{
${ }^{*}$ Corresponding author

Email addresses: mederic.argentina@unice.fr (Médéric Argentina), gerard.iooss@unice.fr (Gérard Iooss)
} 
the linearized operator.

Keywords: Quasipattern, Viscous flows, Faraday instability, Normal forms, Bifurcations

\section{Introduction}

As curator of experiments of the Royal Society, R. Hooke proposed new experiments on a weekly basis. Among these numerous demonstrations, he observed the appearance of a pattern when a rectangular piece of glass recovered with flavor was excited on its edge with a violin bow ${ }^{1}$. This experiment has been largely explored by Chladni in the field of acoustics ${ }^{2}$ and later revisited by Faraday ${ }^{3}$. This latter observed that the response of a vertically forced thin layer of fluid may result in the appearance of waves at the surface with half the frequency forcing. This non trivial result has been criticized at that time by ${ }^{4,5}$ who observed a fluid response with a frequency equal to the forcing one. Later on, Rayleigh ${ }^{6}$ unified these two different observations by proposing a Mathieu equation for describing the instability. The linear analysis of inviscid liquids computed by Benjamin \&Ursell ${ }^{7}$ yielded the sought Mathieu equation for the eigenmodes of vibration. The analysis of the corresponding eigenvalues predicted within a satisfactory agreement the instability threshold in comparison with their experiments, but misses the effects of viscosity. In order to explore the inclusion of viscous stresses, numerical simulations of a thin and viscous layer of fluid vertically forced has been performed ${ }^{8}$ and confronted to various experiments with success ${ }^{9,10}$. For the linear stability analysis, an approximation for viscous liquids has been derived in the form of a damped Mathieu equation ${ }^{11}$ which recovers the numerical approach of ${ }^{8}$. All the previous cited works aimed at predicting the wavelength instability at threshold. Although experiments demonstrate a clear appearance of a well defined characteristic length, the associated patterns at the surface may appear with very different shapes ${ }^{12}$, as a combination of the marginally stable wave vectors. Indeed, this variety is expressed in experiments : from stripes in very viscous systems ${ }^{13}$, squares ${ }^{14,15,16,13}$ and hexagons ${ }^{17,18,19}$. In direct numerical simulations of the viscous fluid layer, these structures have been reproduced ${ }^{20}$. Quasi crystalline structure has been observed when a small container is forced with two frequencies with a twelvefold symmetry in the Fourier Space ${ }^{21}$. Later on, these quasipatterns have been obtained experimentally with just one forcing frequency on large systems ${ }^{22}$. In order 
to predict the pattern selection, analytical approaches has been developed in the approximation of the amplitude equations. These models take the form:

$$
\partial_{t} A_{n}=\mu A_{n}-g_{0} A_{n}^{3}-\sum_{m=1, m \neq n}^{2 q} g\left(\theta_{m n}\right)\left|A_{m}\right|^{2} A_{n}
$$

where $\mu$ measures the distance to the instability threshold, $A_{n}$ is the amplitude of an eigen-mode with a wavevector $\mathbf{k}_{\mathbf{n}}$. The $2 q \geq 8$ vectors $\mathbf{k}_{\mathbf{n}}$ point towards the circle of radius $k_{c}$ and are equally separated by an angle $\pi / q$. When $q$ is not a multiple of 3 , (see section 4 below), the nonlinearities are cubic because of the symmetry $A_{n} \rightarrow-A_{n}$, and are defined through a selfinteraction parameter $g_{0}$ and $g\left(\theta_{m n}\right)$ that couples the amplitudes $A_{m}$ to $A_{n}$. This function has been computed for inviscid fluid ${ }^{23,24,25}$, and exhibits singularities. A criterion of selection of the pattern is given in ${ }^{26}$, relying on an energy argument. Moreover, in ${ }^{26}$, on their model PDE, Rucklidge and Silber study the resonant interactions between critical modes and weakly damped modes, giving another criterion of selection for bifurcating quasipatterns.

The small viscosity limit for an infinite layer of fluid has been addressed in $^{27,28,29}$. The large viscosity limit has been solved linearly in ${ }^{11}$, and the weakly nonlinear analysis performed in ${ }^{30}$. A mechanism for observing quasipatterns have been proposed in ${ }^{13}$ for the two frequency forcing, that is similar to the scenario proposed in ${ }^{26}$ : due to quadratic nonlinearities, a coupling between two sub-harmonic modes produces an harmonic mode that resonates if the harmonic response is marginal, as the sub-harmonic.

In this work, we analyze the scenario for obtaining quasi-crystalline structures, in the approximation of a thin viscous fluid layer. For this limit, ${ }^{31}$ proposes a set of averaged equations based on the lubricated flows of Stokes, that takes into account, as a perturbation, the effect of inertial effects. Since this model is able to reproduce the experimental phase diagrams ${ }^{32}$, it should possess all the necessary nonlinearities to predict the existence of quasipatterns. We present below a new possible mechanism for the natural selection of a quasipattern.

Starting in section 2 from the fluid dynamics equations of ${ }^{31}$, we study the stability, under harmonic vertical forcing oscillations, of the basic uniform state (zero horizontal velocity, flat free surface). Nondimensionalized parameters are the amplitude of the forcing $\Gamma$, the Reynolds number $\mathcal{R}$, and the ratio $\mathcal{B}$ between the Bond number (where surface tension occurs) and the square of the Froude number (where gravity occurs). We consider cases 
where criticallity is obtained with two critical wave numbers $k_{c}$ and $k_{c}^{\prime}$, leading to two critical circles (due to rotational invariance) in the Fourier plane, and we study situations near critical values $\left(\Gamma_{c}, \mathcal{B}_{c}, \mathcal{R}_{c}\right)$ for which the ratio $k_{c} / k_{c}^{\prime}$ corresponds to quadratic interactions of the form

$$
\mathbf{k}_{j}+\mathbf{k}_{l}=\mathbf{k}_{n}^{\prime} \text {, with }\left|\mathbf{k}_{j}\right|=\left|\mathbf{k}_{l}\right|=k_{c},\left|\mathbf{k}_{n}^{\prime}\right|=k_{c}^{\prime}
$$

and the angle $\left(\mathbf{k}_{j}, \mathbf{k}_{l}\right)$ is a multiple of $\pi / q$ (the value of the ratio determines $q$ ). The set of wave vectors $\mathbf{k}_{j}, j=1, \ldots 2 q$, equally spaced on the circle of radius $k_{c}$, generates a $2 q$ - fold quasipattern, as soon as $q \geq 4$. In section 2 we introduce the function spaces required for understanding mathematically quasipatterns, and we study the Floquet exponents of the linearized system, leading to criticallity and allowing at section 3 and in Appendix to define the critical values $\left(\Gamma_{c}, \mathcal{B}_{c}, \mathcal{R}_{c}\right)$. At section 4 we give, for all cases with $q$ odd (the case $q$ even is reported in Appendix), amplitude equations for all solutions invariant under rotations of angle $2 \pi / q$ (twice the angle between the basic wave vectors $\mathbf{k}_{j}$ ). In particular, we show, for $q$ odd, the existence of a quasipattern solution such that a time shift by one period of the forcing, is equivalent to a rotation of the pattern by an angle of $\pi / q$. In addition, we give large families of quasipattern solutions (all invariant under $2 \pi / q$ rotations) and we give necessary conditions for their stability (with respect to perturbations built on the same quasilattice). We compute explicitely the main coefficients of the amplitude equations in two cases with $q=5$ or 7 , checking in such cases that the above necessary conditions are fullfilled.

The existence results given here are not mathematically justified, because of a small divisor problem, not yet solved. This is also the case for our formal stability studies provided for of all bifurcating quasipatterns that we give below.

\section{Basic system and main assumptions}

\subsection{Physical system}

We consider a thin horizontal fluid layer which is vertically harmonically shaked (frequency forcing $\Omega$, only one harmonic). We use the system derived in $^{31}$, where the unknown is the vector $(\eta, \mathbf{v})$ function of $(\mathbf{x}, t), \mathbf{v}$ being the (2 components) average over the vertical of the horizontal component of the velocity of particles, $\eta$ being the elevation of the free surface and $\mathbf{x}$ being the horizontal 2-components coordinate. Notice that the scaling chosen for 
obtaining (2) from the system in ${ }^{31}$ uses a horizontal length scale $L / \sqrt{\mathcal{B} o}$, and velocity scale $\Omega L / \sqrt{\mathcal{B} o}$, where $\mathcal{B} o=\rho \Omega^{2} L^{2} / \gamma h$ is the Bond number $(\rho$ is the density, $\Omega$ is the frequency forcing, $L$ is a characteristic horizontal length, $h$ the thickness of the fluid layer at rest supposed to be very small with respect to $L$, and $\gamma$ is the surface tension). Our system reads as

$$
\begin{aligned}
& \partial_{t} \eta+\nabla \cdot \mathbf{v}=0 \\
& \left(\eta \partial_{t}+\frac{9}{7} \mathbf{v} \cdot \nabla\right) \frac{\mathbf{v}}{\eta}+\frac{1}{7} \frac{\mathbf{v}}{\eta}(\nabla \cdot \mathbf{v})+\frac{5}{2 \mathcal{R}} \frac{\mathbf{v}}{\eta^{2}}+\frac{5 \mathcal{B}}{6} \eta\left(f(t)-\nabla^{2}\right) \nabla \eta=0
\end{aligned}
$$

where the Reynolds number $\mathcal{R}$, defined by $\mathcal{R}=\left(\Omega h^{2}\right) / \nu$, and $\mathcal{B}=\left(\rho L^{2} g\right) / \gamma$ are parameters, where $\nu$ is the kinematic viscosity $(\mathcal{B}$ is the ratio between the Bond number, and the square of the Froude number, where gravity $g$ occurs). The function $f(t)$ is of the form

$$
f(t)=1+\Gamma \cos t
$$

and corresponds to a parametric forcing of frequency 1 (the scale of time is chosen for having this frequency), the parameter $\Gamma$ measures the amplitude of the forcing.

\subsection{Abstract formulation}

We see on system $(2)$, that $(\eta, \mathbf{v})=($ const, $\mathbf{0})$ is a family of equilibria (trivial solutions). In fact, this family corresponds to a change in the definition of the basic depth $h$. This is why we choose to restrict $\eta$ to having a spatial average equal to 1 . Let us define the 3-components vector $U=(\xi, \mathbf{v})$, function of $(\mathbf{x}, t)$ where $\eta=1+\xi$, and then assume that $\xi$ has a zero spatial average. Then (2) may be written as

$$
\frac{d U}{d t}=\mathbf{L}_{\Gamma, \mathcal{B}, \mathcal{R}}(t) U+\mathbf{B}_{\Gamma, \mathcal{B}, \mathcal{R}}(t)(U, U)+\mathbf{C}_{\mathcal{R}}(U, U, U)+O\left(\|U\|^{4}\right)
$$

where all coefficients are $2 \pi$ - periodic in $t$. The linear operator $\mathbf{L}_{\Gamma, \mathcal{B}, \mathcal{R}}(t)$ depending smoothly on parameters $(\Gamma, \mathcal{B}, \mathcal{R})$ is defined by

$$
\mathbf{L}_{\Gamma, \mathcal{B}, \mathcal{R}}(t) U=\left(\begin{array}{c}
-\nabla \cdot \mathbf{v} \\
-\frac{5}{2 \mathcal{R}} \mathbf{v}-\frac{5 \mathcal{B}}{6}(f(t)-\Delta) \nabla \xi
\end{array}\right)
$$


the operator $\mathbf{B}_{\Gamma, \mathcal{B}, \mathcal{R}}(t)$ is quadratic and $\mathbf{C}_{\mathcal{R}}$ is a cubic operator, defined by

$$
\begin{aligned}
\mathbf{B}_{\Gamma, \mathcal{B}, \mathcal{R}}(t)(U, U) & =\left(\begin{array}{c}
0 \\
-\frac{8}{7} \mathbf{v} \nabla \cdot \mathbf{v}-\frac{9}{7} \mathbf{v} \cdot \nabla \mathbf{v}+\frac{5}{\mathcal{R}} \xi \mathbf{v}-\frac{5 \mathcal{B}}{6} \xi\left(f(t)-\nabla^{2}\right) \nabla \xi
\end{array}\right), \\
\mathbf{C}_{\mathcal{R}}(U, U, U) & =\left(\begin{array}{c}
0 \\
\frac{8}{7} \xi \mathbf{v} \nabla \cdot \mathbf{v}+\frac{9}{7} \xi \mathbf{v} \cdot \nabla \mathbf{v}+\frac{9}{7} \mathbf{v}(\mathbf{v} \cdot \nabla \xi)-\frac{15}{2 \mathcal{R}} \xi^{2} \mathbf{v}
\end{array}\right) .
\end{aligned}
$$

All operators act in a Hilbert space $\mathcal{H}$ which corresponds to quasipatterns (see next section). Moreover the system (4) is $O(2)$ equivariant. This means that there is a symmetry $\mathbf{S}$, and a one parameter family of linear operators $\mathbf{R}_{\phi}$ acting in $\mathcal{H}$, representing the rotation of angle $\phi$ in the $\mathbf{x}$ - plane $\mathbb{R}^{2}$, such that

$$
\begin{aligned}
\mathbf{S}^{2} & =\mathbb{I}, \mathbf{S R}_{\phi}=\mathbf{R}_{-\phi} \mathbf{S}, \\
\mathbf{R}_{\phi} \mathbf{R}_{\psi} & =\mathbf{R}_{\phi+\psi}, \mathbf{R}_{0}=\mathbb{I}=\mathbf{R}_{2 \pi}, \forall \phi, \psi \in \mathbb{R}
\end{aligned}
$$

and such that operators $\mathbf{L}_{\Gamma, \mathcal{B}, \mathcal{R}}(t), \mathbf{B}_{\Gamma, \mathcal{B}, \mathcal{R}}(t), \mathbf{C}_{\mathcal{R}}$ commute with $\mathbf{S}$ and $\mathbf{R}_{\phi}$ for any $\phi \in \mathbb{R}$. Operators $\mathbf{S}$ and $\mathbf{R}_{\phi}$ are defined as follows. First the symmetry $\mathbf{S}$ is defined by

$$
\mathbf{S} U(\mathbf{x}, t)=(\xi(\mathbf{s x}, t), \mathbf{s v}(\mathbf{s x}, t)), \text { withsx }=\left(x_{1},-x_{2}\right) .
$$

Let us now introduce operators $R_{\phi}^{\prime}$ acting in $\mathbb{R}^{3}$, defined by

$$
R_{\phi}^{\prime}(\xi, \mathbf{v})=\left(\xi, R_{\phi} \mathbf{v}\right)
$$

where $R_{\phi}$ is the usual rotation of angle $\phi$ in $\mathbb{R}^{2}$, then the operator $\mathbf{R}_{\phi}$ is defined by

$$
\left(\mathbf{R}_{\phi} U\right)(\mathbf{x}, t)=R_{\phi}^{\prime} U\left(R_{-\phi} \mathbf{x}, t\right)=\left(\xi\left(R_{-\phi} \mathbf{x}, t\right), R_{\phi} \mathbf{v}\left(R_{-\phi} \mathbf{x}, t\right)\right)
$$

and we have the group property (7).

\subsection{Main assumptions on the linearized system}

Let us consider the linear system

$$
\frac{d U}{d t}=\mathbf{L}_{\Gamma, \mathcal{B}, \mathcal{R}}(t) U
$$

and look for solutions under the form

$$
U(t, \mathbf{x})=\widehat{U}_{\mathbf{k}}(t) e^{i \mathbf{k} \cdot \mathbf{x}+\sigma t}
$$


where $\mathbf{x}$ and $\mathbf{k} \in \mathbb{R}^{2}$ and $\sigma \in \mathbb{C}$ (Floquet exponent). As we are in the parametric forcing case, we may look for eigenmodes $\widehat{U}_{\mathbf{k}}(t)$ which are $4 \pi / p$ - periodic in $t$ (for example $p=1$ corresponds to frequency $1 / 2, p=2$ to frequency 1, etc..). From the rotational invariance, we have

$$
\mathbf{R}_{\phi} \widehat{U}_{\mathbf{k}}(t) e^{i \mathbf{k} \cdot \mathbf{x}+\sigma t}=R_{\phi}^{\prime} \widehat{U}_{\mathbf{k}}(t) e^{i \mathbf{k} \cdot R_{-\phi} \mathbf{x}+\sigma t}=R_{\phi}^{\prime} \widehat{U}_{\mathbf{k}}(t) e^{i R_{\phi} \mathbf{k} \cdot \mathbf{x}+\sigma t} .
$$

It results that if there is one eigenvector corresponding to the wave vector $\mathbf{k}$ and Floquet exponent $\sigma$, then

$$
R_{\phi}^{\prime} \widehat{U}_{\mathbf{k}}(t)=\widehat{U}_{R_{\phi} \mathbf{k}}(t), \phi \in \mathbb{R}
$$

is also an eigenvector for the wave vector $R_{\phi} \mathbf{k}$, and the full circle of radius $|\mathbf{k}|$ gives a set of eigenvectors for the same Floquet exponent $\sigma$.

We show in Appendix A that for any fixed $|\mathbf{k}|$ and $p$ the Floquet exponents $\sigma$ are such that

$$
\operatorname{Re} \sigma<a_{0 p}(|\mathbf{k}|, \mathcal{B}, \mathcal{R}, \Gamma)
$$

with

$$
a_{0 p}(|\mathbf{k}|, \mathcal{B}, \mathcal{R}, \Gamma)<0 \operatorname{if} \mathcal{R}<5\left(\left(1+|\mathbf{k}|^{2}\right)-\Gamma\right) / \Gamma .
$$

It results that neutral modes corresponding to $\operatorname{Re} \sigma=0$ may be obtained only if $\Gamma=\Gamma_{p}(|\mathbf{k}|, \mathcal{B}, \mathcal{R})$ with

$$
\Gamma_{p}(|\mathbf{k}|, \mathcal{B}, \mathcal{R}) \geq \frac{1+|\mathbf{k}|^{2}}{1+\mathcal{R} / 5} .
$$

Moreover, a perturbation analysis (see Appendix A) shows that for $|\mathbf{k}|$ close to 0 ,

$$
\operatorname{Re} \sigma=-\frac{\mathcal{B R}}{3}|\mathbf{k}|^{2}+O\left(|\mathbf{k}|^{4}\right)<0 .
$$

This implies that the critical value $\Gamma_{c}$ of $\Gamma$, defined by

$$
\min _{|\mathbf{k}|} \Gamma_{p}(|\mathbf{k}|, \mathcal{B}, \mathcal{R})=\Gamma_{c}(p, \mathcal{B}, \mathcal{R})
$$

is reached for $|\mathbf{k}|=k_{c} \neq 0$ where $k_{c}$ depends on $(p, \mathcal{B}, \mathcal{R})$.

We numerically see that for a given $p$ the minimum of $\Gamma_{p}$ is reached in only one point $k_{c}$. Typical pictures are shown in Figure 1.

We now make the following fundamental hypothesis:

Hypothesis 1. In the $(\mathcal{B}, \mathcal{R})$ - space of parameters, there is a set of values given by $q(\mathcal{B}, \mathcal{R})=0$ for which the minimal values for all $\Gamma_{p}(\mathcal{B}, \mathcal{R},|\mathbf{k}|)$, 
$p=1,2, \ldots$ is obtained at the same time for $p=1$ (frequency $1 / 2$ ) with $|\mathbf{k}|=k_{c}$ and for $p=2$ (frequency 1 ) with $\left|\mathbf{k}^{\prime}\right|=k_{c}^{\prime}$. All other $\Gamma_{c}(p, \mathcal{B}, \mathcal{R})$, $p=3,4, \ldots$ are larger.

It results from Hypothesis 1 that we have solutions (10) of (9) (unique up to a factor), where $\sigma=0$, of the form

$$
\begin{aligned}
\boldsymbol{\xi}_{0} & =\widehat{U}_{k_{c}}(t) e^{i \mathbf{k} \cdot \mathbf{x}}, \\
\boldsymbol{\xi}_{1} & =\widehat{U}_{k_{c}^{\prime}}(t) e^{i \mathbf{k}^{\prime} \cdot \mathbf{x}}, \mathbf{k}=\left(k_{c}, 0\right), \mathbf{k}^{\prime}=\left(k_{c}^{\prime}, 0\right), \\
\widehat{U}_{k_{c}}(t+2 \pi) & =-\widehat{U}_{k_{c}}(t) 4 \pi-\text { periodic in } t, \text { while } \widehat{U}_{k_{c}^{\prime}}(t+2 \pi)=\widehat{U}_{k_{c}^{\prime}}(t) .
\end{aligned}
$$

Notice that we have in this case

$$
\left\{\frac{d}{d t}-\mathbf{L}_{\Gamma_{c}, \mathcal{B}, \mathcal{R}}(t)\right\} \boldsymbol{\xi}_{j}=0, j=0,1 .
$$

This means that the set $\left\{\mathbf{R}_{\phi} \boldsymbol{\xi}_{j}, \quad j=0,1\right.$ for all $\left.\phi \in \mathbb{R}\right\}$, is included in the kernel of $\frac{d}{d t}-\mathbf{L}_{\Gamma_{c}, \mathcal{B}, \mathcal{R}}(t)$ acting in the space of $4 \pi$ - time periodic $U$ bounded for $\mathrm{x} \in \mathbb{R}^{2}$.

We define $\mu=\Gamma-\Gamma_{c}$ where $\Gamma_{c}$ is the minimum obtained in Hypothesis 1 (see Figure 1 on the right).

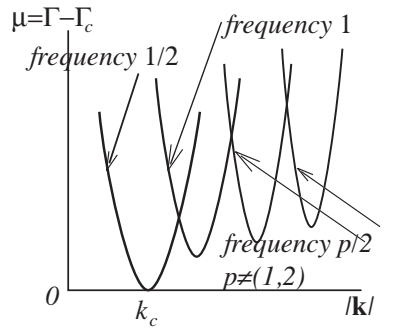

(a)

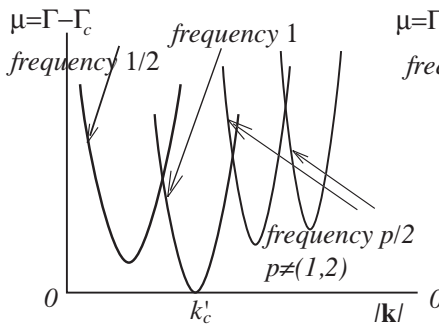

(b)

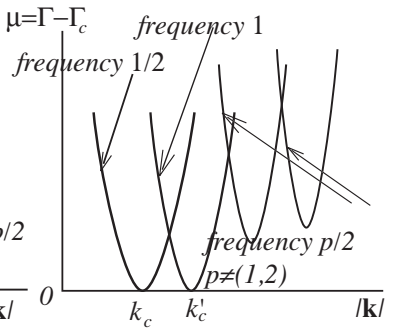

(c)

Figure 1: Neutral stability curves for various values of $(\mathcal{B}, \mathcal{R})$. Hypothesis 1 corresponds to $(c)$.

Remark 1. Hypothesis 1 is satisfied for suitable values of $(\mathcal{B}, \mathcal{R})$ in system (2) as it is numerically shown (see Appendix B). 
(a)

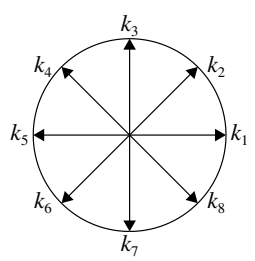

(b)

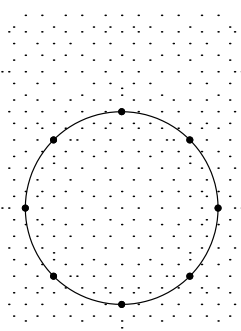

(c)

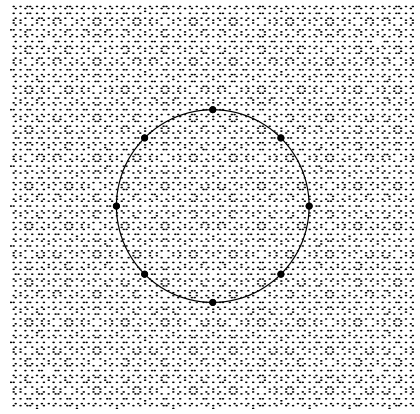

Figure 2: Example quasilattice with $q=4$, after ${ }^{33}$. (a) The 8 wavevectors with $|\mathbf{k}|=1$ that form the basis of the quasilattice. (b,c) The truncated quasilattices $\Sigma_{9}$ and $\Sigma_{27}$. The small dots mark the positions of combinations of up to 9 or 27 of the 8 basis vectors on the unit circle. Note how the density of points increases with $N_{\mathbf{k}}$ (defined in section 2.5).

\subsection{Quasipatterns}

Let $q \geq 4$ be an integer and define wavevectors

$$
\mathbf{k}_{j}=k_{c}\left(\cos \left(\pi \frac{j-1}{q}\right), \sin \left(\pi \frac{j-1}{q}\right)\right), \quad j=1,2, \ldots, 2 q
$$

(see figure 2a). We define the quasilattice $\Sigma \subset \mathbb{R}^{2}$ to be the set of points spanned by integer combinations $\mathbf{k}_{\mathbf{m}}$ of the form

$$
\mathbf{k}_{\mathbf{m}}=\sum_{j=1}^{2 q} m_{j} \mathbf{k}_{j}, \quad \text { where } \quad \mathbf{m}=\left(m_{1}, m_{2}, \ldots, m_{2 q}\right) \in \mathbb{N}^{2 q}
$$

the quasilattice $\Sigma$ is symmetric with respect to the origin and it is known that $\Sigma$ is dense in $\mathbb{R}^{2}$.

Remark 2. Notice that $\mathbf{k}_{j}$ and $-\mathbf{k}_{j}=\mathbf{k}_{j+q}$ belong to the basic set of wave vectors. This implies that if $\mathbf{k} \in \Sigma$, then $-\mathbf{k} \in \Sigma$.

\subsection{Function spaces}

We characterise the functions of interest by their Fourier coefficients on the quasilattice $\Sigma$ generated by the $Q=2 q$ unit vectors $\mathbf{k}_{j}$ :

$$
u(\mathbf{x})=\sum_{\mathbf{k} \in \Sigma} u_{\mathbf{k}} e^{i \mathbf{k} \cdot \mathbf{x}}
$$


where for the moment we assume that $u$ has only one scalar component. Recall that for each $\mathbf{k} \in \Sigma$, there exists a vector $\mathbf{m} \in \mathbb{N}^{2 q}$ such that $\mathbf{k}=$ $\mathbf{k}_{\mathbf{m}}=\sum_{j=1}^{2 q} m_{j} \mathbf{k}_{j}$ and we can choose $\mathbf{m}$ such that $|\mathbf{m}|=\sum_{j=1}^{2 q} m_{j}=N_{\mathbf{k}}$ as defined by

$$
N_{\mathbf{k}}=\min \left\{|\mathbf{m}| ; \mathbf{k}=\mathbf{k}_{\mathbf{m}} \in \Sigma\right\} .
$$

Notice that, since $\mathbf{k}$ and $-\mathbf{k} \in \Sigma$,

$$
\overline{u(\mathbf{x})}=\sum_{\mathbf{k} \in \Sigma} \overline{u_{\mathbf{k}}} e^{-i \mathbf{k} \cdot \mathbf{x}}=\sum_{\mathbf{k} \in \Sigma} \overline{u_{-\mathbf{k}}} e^{i \mathbf{k} \cdot \mathbf{x}}
$$

and $u(\mathbf{x})$ is real valued if $u_{\mathbf{k}}=\overline{u_{-\mathbf{k}}}, \mathbf{k} \in \Sigma$. We have the following properties, proved in ${ }^{12}$ :

Lemma 3. The space of functions

$$
\mathcal{H}_{s}=\left\{u=\sum_{\mathbf{k} \in \Sigma} u_{\mathbf{k}} e^{i \mathbf{k} \cdot \mathbf{x}}:\|u\|_{s}^{2}=\sum_{\mathbf{k} \in \Sigma}\left(1+N_{\mathbf{k}}^{2}\right)^{s}\left|u_{\mathbf{k}}\right|^{2}<\infty\right\},
$$

is a Hilbert space with the scalar product

$$
\langle w, v\rangle_{s}=\sum_{\mathbf{k} \in \Sigma}\left(1+N_{\mathbf{k}}^{2}\right)^{s} w_{\mathbf{k}} \bar{v}_{\mathbf{k}}
$$

Moreover $\mathcal{H}_{s}$ is a Banach algebra for $s>q / 2$. In particular there exists $c_{s}>0$ such that

$$
\|u v\|_{s} \leq c_{s}\|u\|_{s}\|v\|_{s} .
$$

For $\ell \geq 0$ and $s>\ell+q / 2, \mathcal{H}_{s}$ is continuously embedded into $\mathcal{C}^{\ell}$.

Remark 4. In our problem, $U$ is vector valued with 3 components. The adaptation of the above Hilbert space is OK for each component, just replacing in the definition of the scalar product $w_{\mathbf{k}} \bar{v}_{\mathbf{k}}$ by $\left(W_{\mathbf{k}}, V_{\mathbf{k}}\right)$ where $(\cdot, \cdot)$ is the Hermitian scalar product in the vector space $\mathbb{C}^{3}$ of components of $U$, not forgetting that $U_{\mathbf{k}}=\left(\xi_{\mathbf{k}}, \mathbf{v}_{\mathbf{k}}\right)$ is such that $\xi_{\mathbf{0}}=0$ (see section 2.2). Below, we denote this subspace by $\mathbb{H}$. We do not specify $s$ in what follows for the choice of space $\mathbb{H}$ ( $s$ may differ for the $\xi(t)$ component and for the $\mathbf{v}(t)$ components, and is different for the time periodic $U(t)$ in the domain of the linear operator $\mathbf{L}_{\Gamma, \mathcal{B}, \mathcal{R}}(t)$ or in its range. 


\subsection{Basic assumption for a quadratic resonance}

In addition to Hypothesis 1, we assume one of the two following Hypothesis.

Hypothesis 2a. There are two integers $q$ and $n$ such that

$$
k_{c}^{\prime}=2 k_{c} \cos \frac{n \pi}{q} \text {. }
$$

Let us define the following vectors in the plane (see Figure 3)

$$
\begin{aligned}
& \mathbf{k}_{j}=k_{c}\left(\cos \frac{(j-1) \pi}{q}, \sin \frac{(j-1) \pi}{q}\right), \quad j=1, \ldots 2 q, \\
& \mathbf{k}_{j}^{\prime}=k_{c}^{\prime}\left(\cos \frac{(j-1) \pi}{q}, \sin \frac{(j-1) \pi}{q}\right), \quad j=1, \ldots 2 q,
\end{aligned}
$$

then (16) means that we have

$$
\mathbf{k}_{j}+\mathbf{k}_{j+2 n}=\mathbf{k}_{j+n}^{\prime}, \quad j=1, . .2 q .
$$

Hypothesis $\mathbf{2 b}$. There are two integers $q$ and $n$ such that

$$
k_{c}^{\prime}=2 k_{c} \cos \frac{(2 n-1) \pi}{2 q} .
$$

This corresponds to

$$
\mathbf{k}_{j}+\mathbf{k}_{j+2 n-1}=\mathbf{k}_{j+n-1 / 2}^{\prime}, \quad j=1, . .2 q,
$$

with (see Figure 3)

$$
\mathbf{k}_{j+n-1 / 2}^{\prime}=R_{\pi / 2 q} \mathbf{k}_{j+n-1}^{\prime} .
$$

Remark 5. Hypothesis 1 imposes that the critical forcing $\Gamma_{c}(\mathcal{B}, \mathcal{R})$ obtained for $4 \pi$ - periodic and for $2 \pi$ - periodic functions are equal $(\mu=0)$. Hypothesis $2 a$ or $2 b$ imposes in addition a restriction for the ratio $k_{c}^{\prime} / k_{c}$ which gives $q$ and $n$. We show in Appendix $B$ that fixing $k_{c}^{\prime} / k_{c}$, i.e. $n / q$ or $(2 n-1) / 2 q$, fixes the parameters $\mathcal{R}$ and $\mathcal{B}$.

Notice that without (17) or (19) there is no quadratic term in amplitude equations, except in very special cases (where q is a multiple of 3) (see below). The search for quasipatterns works well with only one circle of radius $k_{c}$, and the bifurcation is in general (without quadratic resonances) of pitchfork type (of order $\sqrt{|\mu|}$ ), but with an arbitrary choice for $q$ (see ${ }^{12}$ for example). The interest of our higher codimension case is that $q$ (i.e. the quasipattern) is determined by the choice of parameters. 

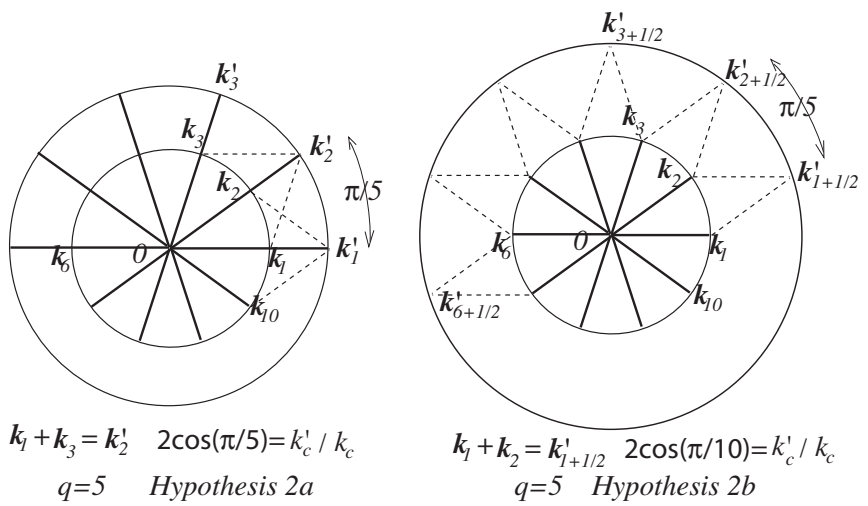

Figure 3: Examples of assumptions on basic wave vectors for $q=5$

\subsection{Kernel and quasi-Fredholm alternative in the $\mathbf{R}_{2 \pi / q}$ rotationally invariant subspace}

Let us consider the kernel of $d / d t-\mathbf{L}_{\Gamma_{c}, \mathcal{B}_{c}, \mathcal{R}_{c}}$ in the space of $4 \pi$ - time periodic functions taking values in $\mathbb{H}_{2 \pi / q}=\left\{U \in \mathbb{H} ; \mathbf{R}_{2 \pi / q} U=U\right\}$. Notice that $2 \pi / q$ is twice the angle between $\mathbf{k}_{j}$ and $\mathbf{k}_{j+1}$. Choosing such a subspace of $\mathbb{H}$ seems somewhat arbitrary. Indeed we might choose to look for all possible solutions in $\mathbb{H}$, or restrict to solutions invariant under $\mathbf{R}_{\pi / q}$ or under any other subgroup of rotations. The purpose here is to give an example of study, not totally trivial, leading to a not too large-dimensional kernel.

From now on, we denote by $\mathbf{L}_{0}$ the linear operator $\mathbf{L}_{\Gamma_{c}, \mathcal{B}_{c}, \mathcal{R}_{c}}$ when hypothesis 1 and $2 \mathrm{a}$ or $2 \mathrm{~b}$ are satisfied.

Because of the rotationnal invariance of (4) which propagates on the linearized system, we can build solutions of the linear system which are invariant under rotations of angle $2 \pi / q$ :

$$
\boldsymbol{\zeta}_{0}(t)=\sum_{j=1}^{q} \widehat{U}_{\mathbf{k}_{2 j-1}}(t) e^{i \mathbf{k}_{2 j-1} \cdot \mathbf{x}}=\sum_{j=1}^{q} \mathbf{R}_{(j-1) 2 \pi / q} \boldsymbol{\xi}_{0}, \quad \mathbf{k}_{2 j-1}=R_{(j-1) 2 \pi / q} \mathbf{k}_{1},
$$

and

$$
\boldsymbol{\zeta}_{1}(t)=\sum_{j=1}^{q} \widehat{U}_{\mathbf{k}_{2 j-1}^{\prime}}(t) e^{i \mathbf{k}_{2 j-1}^{\prime} \cdot \mathbf{x}}=\sum_{j=1}^{q} \mathbf{R}_{(j-1) 2 \pi / q} \boldsymbol{\xi}_{1}, \quad \mathbf{k}_{2 j-1}^{\prime}=R_{(2 j-2) \pi / q} \mathbf{k}_{1}^{\prime},
$$


for Hypothesis 2a, or

$$
\begin{aligned}
\boldsymbol{\zeta}_{1}(t) & =\sum_{j=1}^{q} \widehat{U}_{\mathbf{k}_{2 j-1 / 2}^{\prime}}(t) e^{i \mathbf{k}_{2 j-1 / 2}^{\prime} \cdot \mathbf{x}}=\sum_{j=1}^{q} \mathbf{R}_{(j-1) 2 \pi / q+\pi / 2 q} \boldsymbol{\xi}_{1}, \\
\mathbf{k}_{2 j-1 / 2}^{\prime} & =R_{(j-1) 2 \pi / q} \mathbf{k}_{1+1 / 2}^{\prime},
\end{aligned}
$$

for Hypothesis $2 \mathrm{~b}$. We have clearly $\boldsymbol{\zeta}_{0}, \boldsymbol{\zeta}_{1} \in \mathbb{H}_{2 \pi / q}$ and the following properties hold:

$$
\begin{aligned}
& \left(\frac{d}{d t}-\mathbf{L}_{0}(t)\right) \boldsymbol{\zeta}_{j}=0, \quad j=0,1, \\
& \boldsymbol{\zeta}_{0}(t+2 \pi)=-\boldsymbol{\zeta}_{0}(t), \quad \boldsymbol{\zeta}_{1}(t+2 \pi)=\boldsymbol{\zeta}_{1}(t), \\
& \mathbf{R}_{\pi / q} \boldsymbol{\zeta}_{0}=\overline{\boldsymbol{\zeta}_{0}}, \quad \mathbf{R}_{\pi / q} \boldsymbol{\zeta}_{1}=\overline{\boldsymbol{\zeta}_{1}} \text { when } q \text { is odd, } \\
& \boldsymbol{\zeta}_{0}, \boldsymbol{\zeta}_{1}, \quad \mathbf{R}_{\pi / q} \boldsymbol{\zeta}_{0} \text { and } \mathbf{R}_{\pi / q} \boldsymbol{\zeta}_{1} \text { are real when } q \text { is even, } \\
& \mathbf{S} \boldsymbol{\zeta}_{0}=\boldsymbol{\zeta}_{0}, \quad \mathbf{S R}_{\pi / q} \boldsymbol{\zeta}_{0}=\mathbf{R}_{\pi / q} \boldsymbol{\zeta}_{0} \\
& \mathbf{S} \boldsymbol{\zeta}_{1}=\boldsymbol{\zeta}_{1}, \quad \mathbf{S R}_{\pi / q} \boldsymbol{\zeta}_{1}=\mathbf{R}_{\pi / q} \boldsymbol{\zeta}_{1} \text {, for Hypothesis 2a, } \\
& \mathbf{S} \boldsymbol{\zeta}_{1}=\mathbf{R}_{\pi / q} \boldsymbol{\zeta}_{1}, \quad \mathbf{S R}_{\pi / q} \boldsymbol{\zeta}_{1}=\boldsymbol{\zeta}_{1} \text {, for Hypothesis } 2 \mathrm{~b} \text {. }
\end{aligned}
$$

The kernel of $\frac{d}{d t}-\mathbf{L}_{0}(t)$ is now 4 -dimensional, since it contains $\boldsymbol{\zeta}_{0}, \boldsymbol{\zeta}_{1}$ and $\mathbf{R}_{\pi / q} \boldsymbol{\zeta}_{0}$, and $\mathbf{R}_{\pi / q} \boldsymbol{\zeta}_{1}$, and there is no other independent vector in the kernel.

Let us now solve linear systems of the form

$$
\left(\frac{d}{d t}-\mathbf{L}_{0}(t)\right) U=F
$$

where $F \in \mathbb{H}_{2 \pi / q}$ and we look for solutions $U$ is this space. Equation (24) may be formally solved if $F$ satisfies 4 compatibility conditions

$$
\left\langle F, \boldsymbol{\zeta}_{0}^{*}\right\rangle=\left\langle F, \boldsymbol{\zeta}_{1}^{*}\right\rangle=\left\langle F, \mathbf{R}_{\pi / q} \boldsymbol{\zeta}_{0}^{*}\right\rangle=\left\langle F, \mathbf{R}_{\pi / q} \boldsymbol{\zeta}_{1}^{*}\right\rangle=0
$$

where we defined the scalar product

$$
\langle U, V\rangle=\frac{1}{4 \pi} \int_{0}^{4 \pi}(U(t), V(t))_{\mathcal{H}_{0}} d t
$$

and $(U(t), V(t))_{\mathcal{H}_{0}}$ is the scalar product (take $s=0$ in the definition (14) above) in the space $\mathcal{H}_{0}$. We also use the fact that in $\mathbb{H}_{2 \pi / q}$ the operator $\mathbf{R}_{\pi / q}$ is such that

$$
\mathbf{R}_{\pi / q}^{*}=\mathbf{R}_{-\pi / q}=\mathbf{R}_{\pi / q} .
$$


In (25) $\boldsymbol{\zeta}_{0}^{*}, \mathbf{R}_{\pi / q} \boldsymbol{\zeta}_{0}^{*}$ and $\boldsymbol{\zeta}_{1}^{*}, \mathbf{R}_{\pi / q} \boldsymbol{\zeta}_{1}^{*}$ are solutions, invariant under $\mathbf{R}_{2 \pi / q}$, of the adjoint problem

$$
\left(\frac{d}{d t}+\mathbf{L}_{0}^{*}(t)\right) \boldsymbol{\zeta}_{j}^{*}(t)=0, j=0,1,
$$

with

$$
\begin{aligned}
\boldsymbol{\zeta}_{0}^{*}(t) & =\sum_{1 \leq j \leq q} \widehat{U}_{\mathbf{k}_{2 j-1}}^{*}(t) e^{i \mathbf{k}_{2 j-1} \cdot \mathbf{x}}, \\
\boldsymbol{\zeta}_{1}^{*}(t) & =\sum_{1 \leq j \leq q} \widehat{U}_{\mathbf{k}_{2 j-1}^{\prime}}^{*}(t) e^{i \mathbf{k}_{2 j-1}^{\prime} \cdot \mathbf{x}} \text { for Hypothesis } 2 \mathrm{a}, \\
\boldsymbol{\zeta}_{1}^{*}(t) & =\sum_{1 \leq j \leq q} \widehat{U}_{\mathbf{k}_{2 j-1 / 2}^{\prime}}^{*}(t) e^{i \mathbf{k}_{2 j-1 / 2}^{\prime} \cdot \mathbf{x}}, \text { for Hypothesis } 2 \mathbf{b},
\end{aligned}
$$

and we have the symmetry properties:

$$
\begin{aligned}
& \boldsymbol{\zeta}_{0}^{*}(t+2 \pi)=-\boldsymbol{\zeta}_{0}^{*}(t), \quad \boldsymbol{\zeta}_{1}^{*}(t+2 \pi)=\boldsymbol{\zeta}_{1}^{*}(t) . \\
& \mathbf{R}_{\pi / q} \boldsymbol{\zeta}_{0}^{*}=\overline{\boldsymbol{\zeta}}_{0}^{*}, \quad \mathbf{R}_{\pi / q} \boldsymbol{\zeta}_{1}^{*}=\overline{\boldsymbol{\zeta}}_{1}^{*} \text { when } q \text { is odd, } \\
& \boldsymbol{\zeta}_{0}^{*}, \boldsymbol{\zeta}_{1}^{*}, \quad \mathbf{R}_{\pi / q} \boldsymbol{\zeta}_{0}^{*} \text { and } \mathbf{R}_{\pi / q} \boldsymbol{\zeta}_{1}^{*} \text { are real when } q \text { is even, } \\
& \mathbf{S} \boldsymbol{\zeta}_{0}^{*}=\boldsymbol{\zeta}_{0}^{*}, \quad \mathbf{S R}_{\pi / q} \boldsymbol{\zeta}_{0}^{*}=\mathbf{R}_{\pi / q} \boldsymbol{\zeta}_{0}^{*}, \\
& \mathbf{S} \boldsymbol{\zeta}_{1}^{*}=\boldsymbol{\zeta}_{1}^{*}, \quad \mathbf{S R}_{\pi / q} \boldsymbol{\zeta}_{1}^{*}=\mathbf{R}_{\pi / q} \boldsymbol{\zeta}_{1}^{*}, \text { for Hypothesis } 2 \mathrm{a}, \\
& \mathbf{S} \boldsymbol{\zeta}_{1}^{*}=\mathbf{R}_{\pi / q} \boldsymbol{\zeta}_{1}^{*}, \quad \mathbf{S R}_{\pi / q} \boldsymbol{\zeta}_{1}^{*}=\boldsymbol{\zeta}_{1}^{*}, \text { for Hypothesis } 2 \mathrm{~b} .
\end{aligned}
$$

We assume in addition that we choose the normalization of $\boldsymbol{\zeta}_{j}^{*}(t), j=0,1$ such that

$$
\begin{aligned}
& \left\langle\boldsymbol{\zeta}_{0}, \boldsymbol{\zeta}_{0}^{*}\right\rangle=1, \quad\left\langle\boldsymbol{\zeta}_{0}, \boldsymbol{\zeta}_{1}^{*}\right\rangle=0 \\
& \left\langle\boldsymbol{\zeta}_{1}, \boldsymbol{\zeta}_{0}^{*}\right\rangle=0, \quad\left\langle\boldsymbol{\zeta}_{1}, \boldsymbol{\zeta}_{1}^{*}\right\rangle=1 .
\end{aligned}
$$

Due to the definition of the scalar product in $\mathcal{H}$, we observe that we have

$$
\begin{aligned}
& \left\langle\boldsymbol{\zeta}_{0}, \mathbf{R}_{\pi / q} \boldsymbol{\zeta}_{0}^{*}\right\rangle=0, \quad\left\langle\boldsymbol{\zeta}_{0}, \mathbf{R}_{\pi / q} \boldsymbol{\zeta}_{1}^{*}\right\rangle=0 \\
& \left\langle\boldsymbol{\zeta}_{1}, \mathbf{R}_{\pi / q} \boldsymbol{\zeta}_{0}^{*}\right\rangle=0, \quad\left\langle\boldsymbol{\zeta}_{1}, \mathbf{R}_{\pi / q} \boldsymbol{\zeta}_{1}^{*}\right\rangle=0
\end{aligned}
$$

Remark 6. The compatibility conditions (25) are not sufficient a priori for solving (24). This is not a true Fredholm alternative, because of a small divisor problem in the formal pseudo-inverse of $\frac{d}{d t}-\mathbf{L}_{0}$ in the subspace 
complement of its kernel in $\mathbf{L}^{2}\left(S^{1}, \mathbb{H}_{2 \pi / q}\right)$. In fact the range of $\frac{d}{d t}-\mathbf{L}_{0}$ in $\mathbf{L}^{2}\left(S^{1}, \mathbb{H}_{2 \pi / q}\right)$ is not closed. For obtaining a solution for (24), we need in addition to check that the r.h.s. belongs to the range of $\frac{d}{d t}-\mathbf{L}_{0}$. In the sequel, this is always satisfied once (25) is satisfied, since we deal with $F$ having a finite Fourier expansion.

\section{Computation of the kernel}

\subsection{Kernel of $\frac{d}{d t}-\mathbf{L}_{\Gamma, \mathcal{B}, \mathcal{R}}(t)$}

Let us compute the form of the $4 \pi$ - time periodic solutions $U$ of

$$
\frac{d U}{d t}-\mathbf{L}_{\Gamma, \mathcal{B}, \mathcal{R}}(t) U=\sigma U,
$$

where $\sigma$ is a Floquet exponent (for the period $4 \pi$ ), and where we look for $U(t)$ in $\mathbb{H}$ (quasi-periodic pattern). Equation (27) leads to the system

$$
\begin{aligned}
\frac{d \xi}{d t}+\nabla \cdot \mathbf{v} & =\sigma \xi \\
\frac{d \mathbf{v}}{d t}+\frac{5}{2 \mathcal{R}} \mathbf{v}+\frac{5 \mathcal{B}}{6}(f(t)-\Delta) \nabla \xi & =\sigma \mathbf{v} .
\end{aligned}
$$

This gives

$$
\left(\frac{d}{d t}-\sigma+\frac{5}{4 \mathcal{R}}\right)^{2} \xi-\frac{25}{16 \mathcal{R}^{2}} \xi-\frac{5 \mathcal{B}}{6}(f(t)-\Delta) \Delta \xi=0 .
$$

The Fourier decomposition in $e^{i \mathbf{k} \cdot \mathbf{x}}$ where $\mathbf{k} \in \Sigma$, leads to

$$
\left(\frac{d}{d t}-\sigma+\frac{5}{4 \mathcal{R}}\right)^{2} \widehat{\xi}_{k}-\frac{25}{16 \mathcal{R}^{2}} \widehat{\xi_{k}}+\frac{5 \mathcal{B} k^{2}}{6}\left(f(t)+k^{2}\right) \widehat{\xi_{k}}=0,
$$

where $k^{2}=|\mathbf{k}|^{2}$, and $\widehat{\xi_{k}}$ is $4 \pi$ - periodic in $t$. Since we are looking for the 0 Floquet exponent (corresponds to the kernel of $\frac{d}{d t}-\mathbf{L}_{\Gamma, \mathcal{B}, \mathcal{R}}(t)$ ), we make $\sigma=0$ and the second order differential equation to solve is now (denoting $\left.u=\widehat{\xi}_{k}\right)$

$$
\ddot{u}+\frac{5}{2 \mathcal{R}} \dot{u}+\frac{5 \mathcal{B} k^{2}}{6}\left[1+\Gamma \cos t+k^{2}\right] u=0 .
$$

Notice that taking the curl of the second equation in (28) leads to (for $\sigma=0$ )

$$
\left(\frac{d}{d t}+\frac{5}{2 \mathcal{R}}\right) \operatorname{curl} \mathbf{v}=0,
$$


which, because of time periodicity, gives

$$
\text { curl } \mathbf{v}=0 .
$$

The eigenvector $U$ takes now the following form, for $\mathbf{k} \neq 0$

$$
U=\left(u, \frac{i \mathbf{k}}{k^{2}} \dot{u}\right) e^{i \mathbf{k} \cdot \mathbf{x}}
$$

where $u$ is a $4 \pi$ - periodic solution of (29). We notice the dependancy in $\mathbf{k}$ of the $\mathbf{v}$ component.

For $\mathbf{k}=0$, we impose $\xi_{\mathbf{0}}=0$ by construction, then

$$
\frac{d \mathbf{v}_{\mathbf{0}}}{d t}+\frac{5}{2 \mathcal{R}} \mathbf{v}_{\mathbf{0}}=\mathbf{0}
$$

which does not lead to a $4 \pi$ - periodic solution $\mathbf{v}_{\mathbf{0}}$.

\subsection{Kernel of the adjoint operator $\frac{d}{d t}+\mathbf{L}_{\Gamma, \mathcal{B}, \mathcal{R}}^{*}(t)$}

Using the scalar product (14) we check easily that the adjoint operator $\mathbf{L}_{\Gamma, \mathcal{B}, \mathcal{R}}^{*}$ is defined by

$$
\mathbf{L}_{\Gamma, \mathcal{B}, \mathcal{R}}^{*} U=\left(\begin{array}{c}
\frac{5 \mathcal{B}}{6}(f(t)-\Delta) \nabla \cdot \mathbf{v} \\
-\frac{5}{2 \mathcal{R}} \mathbf{v}+\nabla \xi
\end{array}\right)
$$

and $U$ in the kernel of $\frac{d}{d t}+\mathbf{L}_{\Gamma, \mathcal{B}, \mathcal{R}}^{*}$ satisfies

$$
\begin{aligned}
\frac{d \xi}{d t}+\frac{5 \mathcal{B}}{6}(f(t)-\Delta) \nabla \cdot \mathbf{v} & =0 \\
\frac{d \mathbf{v}}{d t}-\frac{5}{2 \mathcal{R}} \mathbf{v}+\nabla \xi & =0
\end{aligned}
$$

Here again $\operatorname{curl} \mathbf{v}=0$, and a coefficient $\left(\widehat{\xi_{k}}, \widehat{\mathbf{v}}\right)$ of $e^{i \mathbf{k} \cdot \mathbf{x}}$ in the Fourier decomposition of $U$ should verify (we use $\nabla(\nabla \cdot \mathbf{v})=\Delta \mathbf{v}$ )

$$
\begin{gathered}
\left(\frac{5}{2 \mathcal{R}} v-\dot{v}, i \mathbf{k} v\right)=\left(\widehat{\xi_{k}}, \widehat{\mathbf{v}}\right) \\
\frac{d^{2} v}{d t^{2}}-\frac{5}{2 \mathcal{R}} \frac{d v}{d t}+\frac{5 \mathcal{B} k^{2}}{6}\left[1+\Gamma \cos t+k^{2}\right] v=0
\end{gathered}
$$

where $v$ is $4 \pi$ - periodic in $t$. Notice that $v$ needs to be scaled for the verification of normalization condition (26). 


\section{Formal derivation and study of the amplitudes equations}

Let us proceed as if we could apply the center manifold reduction (this is not true indeed!) for each of the cases we consider. In fact when we compute the Taylor expansion of a center manifold here, we are faced with a small divisor problem for large orders in the expansion. We decide below to ignore this fact, but this has the consequence that we cannot derive reduced equations (amplitude equations given below) on a center manifold up to any orders.

Let us denote the parameter set by $(\mu, \beta, \gamma)=\left(\Gamma-\Gamma_{c}, \mathcal{B}-\mathcal{B}_{c}, \mathcal{R}-\mathcal{R}_{c}\right)$ and define the $2 \pi-t$-periodic linear operators $\mathbf{L}_{j}, j=1,2,3$ by

$$
\begin{aligned}
& \mathbf{L}_{\Gamma, \mathcal{B}, \mathcal{R}}=\mathbf{L}_{0}+\mu \mathbf{L}_{1}+\beta \mathbf{L}_{2}+\gamma \mathbf{L}_{3}+O\left\{(|\mu|+|\beta|+\gamma \mid)^{2}\right\} \\
& \mathbf{L}_{1}(t) U=\left(\begin{array}{c}
0 \\
-\frac{5 \mathcal{B}_{c}}{6} \nabla \xi \cos t
\end{array}\right), \mathbf{L}_{3} U=\left(\begin{array}{c}
0 \\
\frac{5}{2 \mathcal{R}_{c}^{2}} \mathbf{v}
\end{array}\right), \\
& \mathbf{L}_{2}(t) U=\left(\begin{array}{c}
0 \\
-\frac{5}{6}\left(1+\Gamma_{c} \cos t-\Delta\right) \nabla \xi
\end{array}\right),
\end{aligned}
$$

and let us define $\mathbf{B}_{0}(t)=\mathbf{B}_{\Gamma_{c}, \mathcal{B}_{c}, \mathcal{R}_{c}}(t)$.

A "center manifold reduction" means that for $(\mu, \beta, \gamma)$ close to 0 , the "small" solutions in $\mathbb{H}_{2 \pi / q}$ that we might observe as $t \rightarrow \infty$ should satisfy

$$
\begin{aligned}
U(t)= & A_{0}(t) \boldsymbol{\zeta}_{0}(t)+A_{1}(t) \boldsymbol{\zeta}_{1}(t)+B_{0}(t) \mathbf{R}_{\pi / q} \boldsymbol{\zeta}_{0}(t)+B_{1}(t) \mathbf{R}_{\pi / q} \boldsymbol{\zeta}_{1}(t)+ \\
& +\boldsymbol{\Psi}\left(A_{0}(t), A_{1}(t), B_{0}(t), B_{1}(t), \mu, \beta, \gamma, t\right)
\end{aligned}
$$

where

$$
\left\langle\Psi, \boldsymbol{\zeta}_{0}^{*}\right\rangle=\left\langle\boldsymbol{\Psi}, \boldsymbol{\zeta}_{1}^{*}\right\rangle=\left\langle\boldsymbol{\Psi}, \mathbf{R}_{\pi / q} \boldsymbol{\zeta}_{0}^{*}\right\rangle=\left\langle\boldsymbol{\Psi}, \mathbf{R}_{\pi / q} \boldsymbol{\zeta}_{1}^{*}\right\rangle=0,
$$

and $\boldsymbol{\Psi}\left(A_{0}, A_{1}, B_{0}, B_{1}, \mu, \beta, \gamma, t\right)$ is $4 \pi$ - periodic in $t$.

Thanks to the symmetry properties (23), in the case when $q$ is odd, we have

$$
B_{0}=\overline{A_{0}}, \quad B_{1}=\overline{A_{1}},
$$

while, when $q$ is even $A_{0}, B_{0}, A_{1}, B_{1}$ are real valued. Now, the time shift of $2 \pi$ changes $\boldsymbol{\zeta}_{0}$ and $\boldsymbol{\zeta}_{0}^{*}$ into their opposites, so that this leads to

$$
\boldsymbol{\Psi}\left(A_{0}, A_{1}, B_{0}, B_{1}, \mu, \beta, \gamma, t+2 \pi\right)=\Psi\left(-A_{0}, A_{1},-B_{0}, B_{1}, \mu, \beta, \gamma, t\right),
$$


and by construction of the center manifold and of the properties (33) for obtaining amplitude on a center manifold, we may check that it is such that

$$
\left(\frac{d A_{0}}{d t}, \frac{d A_{1}}{d t}, \frac{d B_{0}}{d t}, \frac{d B_{1}}{d t}\right)=\left(f_{0}, f_{1}, g_{0}, g_{1}\right)\left(A_{0}, A_{1}, B_{0}, B_{1}, \mu, \beta, \gamma, t\right)
$$

with $2 \pi$ - periodic in $t$ functions $f_{0}, f_{1}, g_{0}, g_{1}$ (instead of $4 \pi$ - periodic), $f_{0}$ and $g_{0}$ being odd in $\left(A_{0}, B_{0}\right)$, while $f_{1}$ and $g_{1}$ are even in $\left(A_{0}, B_{0}\right)$.

\subsection{Amplitude equations for $q$ odd}

We put in Appendices Appendix $\mathrm{C}$ and Appendix D the amplitude equations for $q$ even, and the study of their solutions.

When $q$ is odd in Hypothesis $2 \mathrm{a}$ or $2 \mathrm{~b}$, we need to satisfy the following symmetry properties (see $(23))$ :

$$
\begin{aligned}
\boldsymbol{\Psi}\left(A_{0}, A_{1}, \overline{A_{0}}, \overline{A_{1}}, \mu, \beta, \gamma, t+2 \pi\right) & =\boldsymbol{\Psi}\left(-A_{0}, A_{1}, \overline{A_{0}}, \overline{A_{1}}, \mu, \beta, \gamma, t\right), \\
\mathbf{R}_{\pi / q} \boldsymbol{\Psi}\left(A_{0}, A_{1}, \overline{A_{0}}, \overline{A_{1}}, \mu, \beta, \gamma, t\right) & =\boldsymbol{\Psi}\left(\overline{A_{0}}, \overline{A_{1}}, A_{0}, A_{1}, \mu, \beta, \gamma, t\right),
\end{aligned}
$$

and the system of amplitude equations satisfied by $\left(A_{0}, A_{1}, \overline{A_{0}}, \overline{A_{1}}\right)$ needs to commute with the actions

$$
\begin{aligned}
& \left(A_{0}, A_{1}, \overline{A_{0}}, \overline{A_{1}}, t\right) \rightarrow\left(-A_{0}, A_{1}, \overline{A_{0}}, \overline{A_{1}}, t\right), \\
& \left(A_{0}, A_{1}, \overline{A_{0}}, \overline{A_{1}}, t\right) \rightarrow\left(\overline{A_{0}}, \overline{A_{1}}, A_{0}, A_{1}, t\right),
\end{aligned}
$$

representing respectively the time shift $t \rightarrow t+2 \pi$ and the rotation $R_{\pi / q}$, and, because of the non trivial action of $\mathbf{S}$ in case of Hypothesis $2 \mathrm{~b}$ :

$$
\left(A_{0}, A_{1}, \overline{A_{0}}, \overline{A_{1}}, t\right) \rightarrow\left(A_{0}, \overline{A_{1}}, \overline{A_{0}}, A_{1}, t\right) .
$$

It is easy to show that the commutation property with $\mathbf{R}_{\pi / q}$ implies that the coefficients of the amplitude equations are real, and the time shift $t \rightarrow t+2 \pi$ invariance leads to oddness in $\left(A_{0}, \overline{A_{0}}\right)$ for the Taylor expansion terms of the r.h.s. of $d A_{0} / d t$, while it leads to evenness in $\left(A_{0}, \overline{A_{0}}\right)$ for the Taylor expansion terms of the r.h.s. of $d A_{1} / d t$.

Moreover we need to keep in mind that the component $d A_{0} / d t$ results from a scalar product with $\boldsymbol{\zeta}_{0}^{*}(t)$, i.e. with Fourier components of the form $e^{i \mathbf{k}_{2 j-1} \cdot \mathbf{x}}$ with $1 \leq j \leq q$, while the component $d A_{1} / d t$ results from a scalar product with $\boldsymbol{\zeta}_{1}^{*}(t)$, i.e. with Fourier components of the form $e^{i \mathbf{k}_{2 j-1}^{\prime} \cdot \mathbf{x}}$ with $1 \leq j \leq q$ for Hypothesis $2 \mathrm{a}$, or of the form $e^{i \mathbf{k}_{2 j-1 / 2}^{\prime} \cdot \mathbf{x}}$ with $1 \leq j \leq q$ for Hypothesis 2b. 
We have for Hypothesis $2 a$

$$
\mathbf{k}_{j}+\mathbf{k}_{j+2 n}=\mathbf{k}_{j+n}^{\prime}
$$

and quadratic resonances occur when $\mathbf{k}_{1}=\mathbf{k}_{1 \pm n}^{\prime}+\mathbf{k}_{1 \pm 2 n+q}$ for $\frac{d A_{0}}{d t}$, and $\mathbf{k}_{1}^{\prime}=\mathbf{k}_{1-n}+\mathbf{k}_{1+n}$ for $\frac{d A_{1}}{d t}$. The form of the system of amplitude equations then depends on the parity of $n$. We obtain at cubic order for Hypothesis $2 a$ with $q$ odd

i) for $n$ odd

$$
\begin{aligned}
& \frac{d A_{0}}{d t}=\mu_{0} A_{0}+a_{0} \overline{A_{0} A_{1}}+b_{0} A_{0}^{2} \overline{A_{0}}+c_{0} A_{0} A_{1} \overline{A_{1}} \\
& \frac{d A_{1}}{d t}=\mu_{1} A_{1}+a_{1}{\overline{A_{0}}}^{2}+b_{1} A_{0} \overline{A_{0}} A_{1}+c_{1} A_{1}^{2} \overline{A_{1}}
\end{aligned}
$$

where the linear coefficients may be expressed as

$$
\begin{aligned}
& \mu_{0}=\alpha_{0} \mu+\beta_{0} \beta+\gamma_{0} \gamma \\
& \mu_{1}=\alpha_{1} \mu+\beta_{1} \beta+\gamma_{1} \gamma
\end{aligned}
$$

with $\alpha_{j}, \beta_{j}, \gamma_{j}, j=0,1$ real. Coefficients $\mu_{j}, a_{j}, b_{j}, c_{j} j=0,1$, are real , $\mu_{0}, \mu_{1}$ being close to 0 .

Remark 7. Higher order terms in (36) are of the form

$$
\begin{aligned}
& A_{0} P\left(\left|A_{0}\right|^{2},\left|A_{1}\right|^{2}, A_{1} A_{0}^{2}, \overline{A_{1}} \overline{A_{0}^{2}}\right)+\overline{A_{0} A_{1}} Q\left(\left|A_{1}\right|^{2}, \overline{A_{1}} \overline{A_{0}^{2}}\right) \\
& A_{1} P^{\prime}\left(\left|A_{0}\right|^{2},\left|A_{1}\right|^{2}, A_{1} A_{0}^{2}, \overline{A_{1}} \overline{A_{0}^{2}}\right)+{\overline{A_{0}}}^{2} Q^{\prime}\left(\left|A_{0}\right|^{2}, \overline{A_{1}} \overline{A_{0}^{2}}\right)
\end{aligned}
$$

where $P, P^{\prime}, Q$ and $Q^{\prime}$ are polynomials in their arguments. However, at some step we obtain very large coefficients of monomials due to the occurence of $e^{i \mathbf{k} \cdot \mathbf{x}}$ with $\mathbf{k}$ very close to $\mathbf{k}_{j}$ or $\mathbf{k}_{j}^{\prime}$ in the Fourier expansion of $d U / d t$ in $\mathbb{H}$. This is due to the fact that the center manifold reduction does not apply here. So, we stop the writing of the normal form at cubic order to avoid this small divisor problem, considering that higher order terms completing this system should satisfy the symmetry invariances (35), having $2 \pi$ - periodic in time coefficients.

ii) For $n$ even we obtain in the same way

$$
\begin{aligned}
& \frac{d A_{0}}{d t}=\mu_{0} A_{0}+a_{0} \overline{A_{0}} A_{1}+b_{0} A_{0}^{2} \overline{A_{0}}+c_{0} A_{0} A_{1} \overline{A_{1}} \\
& \frac{d A_{1}}{d t}=\mu_{1} A_{1}+a_{1} A_{0}^{2}+b_{1} A_{0} \overline{A_{0}} A_{1}+c_{1} A_{1}^{2} \overline{A_{1}}
\end{aligned}
$$


all coefficients $\mu_{0}, \mu_{1}, a_{j}, b_{j}, c_{j}$ being real, $\mu_{0}, \mu_{1}$ being close to 0 .

Now for Hypothesis $2 b$, we have

$$
\mathbf{k}_{j}+\mathbf{k}_{j+2 n-1}=\mathbf{k}_{j+n-1 / 2}^{\prime}
$$

and quadratic resonances occur when $\mathbf{k}_{1}=\mathbf{k}_{n+1 / 2}^{\prime}+\mathbf{k}_{2 n+q}$ and $\mathbf{k}_{1}=\mathbf{k}_{1-n+1 / 2}^{\prime}+$ $\mathbf{k}_{2-2 n+q}$ for $\frac{d A_{0}}{d t}$, and when $\mathbf{k}_{1+1 / 2}^{\prime}=\mathbf{k}_{2-n}+\mathbf{k}_{2+n-1}$ for $\frac{d A_{1}}{d t}$, which leads to (using the action of $\mathbf{S}$ )

$$
\begin{aligned}
\frac{d A_{0}}{d t} & =\mu_{0} A_{0}+a_{0} A_{0}\left(A_{1}+\overline{A_{1}}\right)+b_{0} A_{0}^{2} \overline{A_{0}}+c_{0} A_{0} A_{1} \overline{A_{1}} \\
\frac{d A_{1}}{d t} & =\mu_{1} A_{1}+a_{1} A_{0} \overline{A_{0}}+b_{1} A_{0} \overline{A_{0}} A_{1}+c_{1} A_{1}^{2} \overline{A_{1}}
\end{aligned}
$$

where $\mu_{j}, a_{j}, b_{j}, c_{j} j=0,1$, are real, $\mu_{0}, \mu_{1}$ being close to 0 .

\subsection{Equilibrium solutions and their stability in the case $q$ odd}

4.2.1. Monomodal solutions of (36), (37) and (38)

The first non trivial equilibrium solution is given by

$$
\begin{aligned}
A_{0} & =0 \\
\mu_{1}+c_{1}\left|A_{1}\right|^{2} & =0 .
\end{aligned}
$$

This is a one parameter family of quasipattern solutions (built with the wave vectors $\mathbf{k}_{j}^{\prime}$ or $\left.\mathbf{k}_{j+1 / 2}^{\prime}\right)$. The study of the linearized system from (36) or from (37) leads to the eigenvalues

$$
0,-2 \mu_{1}, \mu_{0} \pm a_{0}\left|A_{1}\right|+c_{0}\left|A_{1}^{2}\right|
$$

so that a necessary condition for the existence and stability of such solutions is

$$
\mu_{1}>0, c_{1}<0, \mu_{0}<\left|a_{0}\right| \sqrt{\frac{\mu_{1}}{-c_{1}}} .
$$

At this order, these solutions form a circle due to the undeterminacy on the phase of $A_{1}$. It is clear that $A_{0}=0$ is still solution at any order, since this corresponds to a quasipattern built with only the lattice spanned by the integer combinations of wave vectors $\mathbf{k}_{j}^{\prime}$ or $\mathbf{k}_{j+1 / 2}^{\prime}$, with no chance to find any $\mathbf{k}_{j}$ in these combinations. Higher order terms in the amplitude equation would generically provide a $2 \pi$ - periodic perturbation of the previous solutions, selecting the phase of $A_{1}$. Our interpretation is that this means that after a certain order, higher order terms (which are not in normal form) in the amplitude equation are used to kill the degeneracy given by the solution for the normal form. 


\subsubsection{Bimodal solutions for $q$ odd and Hypothesis 2 a}

To fix ideas, let us consider the case with $n$ odd (see (36)) and define polar coordinates

$$
A_{0}=r_{0} e^{i \theta_{0}}, A_{1}=r_{1} e^{i \theta_{1}}
$$

and

$$
\Theta=2 \theta_{0}+\theta_{1}
$$

so that the system (36) becomes

$$
\begin{aligned}
\frac{d r_{0}}{d t} & =\left(\mu_{0}+a_{0} r_{1} \cos \Theta+b_{0} r_{0}^{2}+c_{0} r_{1}^{2}\right) r_{0}, \\
\frac{d \theta_{0}}{d t} & =-a_{0} r_{1} \sin \Theta, \\
\frac{d r_{1}}{d t} & =\left(\mu_{1}+b_{1} r_{0}^{2}+c_{1} r_{1}^{2}\right) r_{1}+a_{1} r_{0}^{2} \cos \Theta \\
\frac{d \theta_{1}}{d t} & =-a_{1} \frac{r_{0}^{2}}{r_{1}} \sin \Theta,
\end{aligned}
$$

with

$$
\frac{d \Theta}{d t}=-\left(a_{1} \frac{r_{0}^{2}}{r_{1}}+2 a_{0} r_{1}\right) \sin \Theta .
$$

Remark 8. For $n$ even, we need to set $\Theta=2 \theta_{0}-\theta_{1}$ and change $a_{1}$ into $-a_{1}$.

Equilibria of type 1. A family of equilibria occurs for $\Theta=0$ or $\pi$. Namely $\sin \Theta=0$ and $\cos \Theta= \pm 1$. We need to solve

$$
\begin{aligned}
\mu_{0} \pm a_{0} r_{1}+b_{0} r_{0}^{2}+c_{0} r_{1}^{2} & =0 \\
\left(\mu_{1}+b_{1} r_{0}^{2}+c_{1} r_{1}^{2}\right) r_{1} \pm a_{1} r_{0}^{2} & =0 .
\end{aligned}
$$

This leads to the existence condition:

$$
\mu_{0} \mu_{1} a_{0} a_{1}>0
$$

and

$$
\begin{aligned}
& r_{0}^{2}=\frac{\mu_{0} \mu_{1}}{a_{0} a_{1}}+O\left(\left(\left|\mu_{0}\right|+\left|\mu_{1}\right|\right)^{3}\right), \\
& r_{1}=\mp \frac{\mu_{0}}{a_{0}} \mp\left(\frac{c_{0} \mu_{0}^{2}}{a_{0}^{3}}+\frac{b_{0} \mu_{0} \mu_{1}}{a_{0}^{2} a_{1}}\right)+O\left(\left(\left|\mu_{0}\right|+\left|\mu_{1}\right|\right)^{3}\right) .
\end{aligned}
$$


The stability of such one-parameter families of solutions (the two possible shifts on $\theta_{0}$ and $\theta_{1}$ are linked by $2 \theta_{0}+\theta_{1}$ being fixed) is given at main order by the eigenvalues of the matrix

$$
\left(\begin{array}{ccc}
2 b_{0} r_{0}^{2} & \pm a_{0} r_{0}+2 c_{0} r_{0} r_{1} & 0 \\
\pm 2 a_{1} r_{0}+2 b_{1} r_{0} r_{1} & \mp a_{1} \frac{r_{0}^{2}}{r_{1}}+2 c_{1} r_{1}^{2} & 0 \\
0 & 0 & 2 \mu_{0}+\mu_{1}
\end{array}\right)
$$

It we notice that $\mp a_{1} \frac{r_{0}^{2}}{r_{1}}=\mu_{1}+O\left(\left(\left|\mu_{0}\right|+\left|\mu_{1}\right|\right)^{2}\right)$, and $2 a_{0} a_{1} r_{0}^{2}=2 \mu_{0} \mu_{1}+$ $O\left(\left(\left|\mu_{0}\right|+\left|\mu_{1}\right|\right)^{3}\right)$, it is then clear that a necessary condition (at main order) for the stability of these families of solutions is

$$
\mu_{1}<-2 \mu_{0}<0
$$

while we need to satisfy

$$
a_{0} a_{1}<0
$$

Physical interpretation of these solutions: We observe that in the above families, if we choose $\theta_{0}=0$ or $\pi$ and $\theta_{1}=0$ or $\pi$, the action of the rotation $\mathbf{R}_{\pi / q}$ is the identity, so that we obtain a one parameter family of solutions invariant under $\mathbf{R}_{\pi / q}$.

If we choose $\theta_{0}=\pi / 2$ or $3 \pi / 2$ and $\theta_{1}=0$ or $\pi$, the actions of the rotation $\mathbf{R}_{\pi / q}$ and of the time shift by $2 \pi$ (period of the forcing) are the same since $A_{0} \rightarrow-A_{0}=\overline{A_{0}}$, while $A_{1}$ is unchanged (real). This means that after the period $2 \pi$ the solution looks like rotated by an angle of $\pi / q$. This type of solution should be more visible if $\left|\mu_{0}\right|$ is small and $\left|\mu_{1}\right|$ quite large, in such a way that the mode $A_{0}$ dominates the mode $A_{1}$.

Other solutions with $2 \theta_{0}+\theta_{1}=0$ or $\pi$ and $\theta_{0} \neq 0, \pi / 2, \pi, 3 \pi / 2$ are such that $\mathbf{R}_{\pi / q}$ acts non trivially, and differently than the time shift by $2 \pi$ which exchanges two different solutions (adds $\pi$ to $\theta_{0}$ ).

Remark 9. The solutions above satisfy $2 \theta_{0}+\theta_{1}=0$ or $\pi$ (modulo $2 \pi$ ). This means that at this order there is a circle of solutions. However the undeterminacy should be eliminated by higher order terms. Indeed, keeping in mind the symmetry (35) of the amplitude system at higher orders, all monomials occur with real coefficients and when $A_{0}, A_{1}$ are written in polar coordinates, it remains powers of $r_{0}$ and $r_{1}$ with factors $\cos \left(m \theta_{0}+p \theta_{1}\right)$ in radial equations, and $\sin \left(m \theta_{0}+p \theta_{1}\right)$ in angular equations, with $m$ even. Since $2 \theta_{0}+\theta_{1}=0$ or $\pi$ (modulo $2 \pi$ ), there are isolated equilibrium solution 
given by the angular equations: $\theta_{1}=0$ or $\pi$ which cancel all terms of the form $\sin \left(m \theta_{0}+p \theta_{1}\right)$. Finally we recover the only possible values for $\theta_{0}$ : $0, \pi / 2, \pi, 3 \pi / 2$, which eliminates the undeterminacy on the phase. It should be noticed that the necessary conditions for the stability (40) are valid for all these solutions. However, since it remains one Floquet exponent close to 0 which is not determined at this step, we cannot concude for this (formal) stability.

Equilibria of type 2. Another type of equilibria occur when

$$
\begin{aligned}
a_{1} r_{0}^{2}+2 a_{0} r_{1}^{2} & =0 \\
\mu_{0}+a_{0} r_{1} \cos \Theta+b_{0} r_{0}^{2}+c_{0} r_{1}^{2} & =0 \\
\left(\mu_{1}+b_{1} r_{0}^{2}+c_{1} r_{1}^{2}\right) r_{1}+a_{1} r_{0}^{2} \cos \Theta & =0 .
\end{aligned}
$$

This system leads to solutions under the first condition

$$
a_{0} a_{1}<0
$$

Then

$$
\begin{aligned}
r_{1}^{2} & =-\frac{a_{1}}{\alpha}\left(\mu_{1}+2 \mu_{0}\right), \\
r_{0}^{2} & =\frac{2 a_{0}}{\alpha}\left(\mu_{1}+2 \mu_{0}\right), \\
r_{1} \cos \Theta & =\frac{-1}{a_{0} \alpha}\left[\left(2 a_{0} b_{0}-a_{1} c_{0}\right) \mu_{1}+\left(a_{1} c_{1}-2 a_{0} b_{1}\right) \mu_{0}\right],
\end{aligned}
$$

with

$$
\alpha=a_{1}\left(2 c_{0}+c_{1}\right)-2 a_{0}\left(2 b_{0}+b_{1}\right) .
$$

We need in addition to check that $|\cos \Theta| \leq 1$, which leads to the condition

$$
\left[\left(2 a_{0} b_{0}-a_{1} c_{0}\right) \mu_{1}+\left(a_{1} c_{1}-2 a_{0} b_{1}\right) \mu_{0}\right]^{2}+\alpha a_{0}^{2} a_{1}\left(\mu_{1}+2 \mu_{0}\right) \leq 0,
$$

which corresponds to the interior region of a parabola $\mathcal{P}$ (tangent to the line $\mu_{1}+2 \mu_{0}=0$ at the origin) in the $\left(\mu_{0}, \mu_{1}\right)$ plane. It should be noticed that these equilibria correspond to periodic solutions of system (36), with $2 \theta_{0}(t)+\theta_{1}(t)$ fixed. Hence, they correspond to a family of solutions of $(2)$ which are time quasi-periodic, in addition to be quasiperiodic in space. 
Remark 10. Equilibria of type 2 are distinct from those of type 1 for $\left(\mu_{0}, \mu_{1}\right)$ not belonging to the parabola $\mathcal{P}$

$$
\left[\left(2 a_{0} b_{0}-a_{1} c_{0}\right) \mu_{1}+\left(a_{1} c_{1}-2 a_{0} b_{1}\right) \mu_{0}\right]^{2}+\alpha a_{0}^{2} a_{1}\left(\mu_{1}+2 \mu_{0}\right)=0,
$$

for which $\cos \Theta= \pm 1$ and are steady equilibria of system (36).

We might give as above necessary conditions for the stability of this family of quasiperiodic solutions, in studying the real part of the 3 eigenvalues of the linearized system in $\left(r_{0}, r_{1}, \Theta\right)$. Since this corresponds to a stability study of a circle of quasiperiodic solutions which is a complicate thing, we do not make the analysis here.

\subsubsection{Bimodal solutions for $q$ odd and Hypothesis $2 b$}

Writting again in polar coordinates, we obtain from (38)

$$
\begin{aligned}
& \frac{d r_{0}}{d t}=\mu_{0} r_{0}+2 a_{0} r_{0} r_{1} \cos \theta_{1}+b_{0} r_{0}^{3}+c_{0} r_{0} r_{1}^{2}, \\
& \frac{d \theta_{0}}{d t}=0, \\
& \frac{d r_{1}}{d t}=\mu_{1} r_{1}+a_{1} r_{0}^{2} \cos \theta_{1}+b_{1} r_{0}^{2} r_{1}+c_{1} r_{1}^{3}, \\
& \frac{d \theta_{1}}{d t}=-a_{1} \frac{r_{0}^{2}}{r_{1}} \sin \theta_{1},
\end{aligned}
$$

which leads to

$$
\begin{aligned}
\theta_{0} \text { undetermined, } \quad \theta_{1} & =0 \text { or } \pi, \\
\mu_{0} \pm 2 a_{0} r_{1}+b_{0} r_{0}^{2}+c_{0} r_{1}^{2} & =0, \\
\pm a_{1} r_{0}^{2}+r_{1}\left(\mu_{1}+b_{1} r_{0}^{2}+c_{1} r_{1}^{2}\right) & =0 .
\end{aligned}
$$

The solutions exist provided that

$$
\mu_{0} \mu_{1} a_{0} a_{1}>0
$$

and

$$
r_{0}^{2}=\frac{\mu_{0} \mu_{1}}{2 a_{0} a_{1}}+\text { h.o.t, } \quad r_{1}=\mp \frac{\mu_{0}}{2 a_{0}}+\text { h.o.t. }
$$

As before, a necessary condition for the stability of any solution in this one parameter family, is that

$$
\mu_{1}<0<\mu_{0}, a_{0} a_{1}<0 .
$$


We have the same discussion as for Hypothesis 2a about solutions with $\theta_{0}=0, \pi / 2, \pi$ or $3 \pi / 2$ which are either invariant under the rotation $\mathbf{R}_{\pi / q}$ or transformed by this rotation into the time shifted (by $2 \pi$ ) solution.

\section{Appendix A. Estimates on critical values}

Let us consider a solution of the form (10) for the system (9), then we obtain

$$
\begin{aligned}
\widehat{U}_{\mathbf{k}}(t)=\left(\xi_{\mathbf{k}}(t), \mathbf{v}_{\mathbf{k}}(t)\right), \text { with } \xi_{\mathbf{0}}=0, \text { and } \xi_{\mathbf{k}}, \mathbf{v}_{\mathbf{k}} 4 \pi / p-\text { periodic } \\
\dot{\xi}_{\mathbf{k}}+\sigma \xi_{\mathbf{k}}+i \mathbf{k} \cdot \mathbf{v}_{\mathbf{k}}=0 \\
\dot{\mathbf{v}}_{\mathbf{k}}+\sigma \mathbf{v}_{\mathbf{k}}+\frac{5}{2 \mathcal{R}} \mathbf{v}_{\mathbf{k}}+\frac{5 \mathcal{B}}{6} i \mathbf{k}\left(f(t)+|\mathbf{k}|^{2}\right) \xi_{\mathbf{k}}=0
\end{aligned}
$$

First we notice the solution for $\mathbf{k}=0$ : since $\xi_{0}=0$ in our subspace $\mathbb{H}$, we have

$$
\dot{\mathbf{v}}_{\mathbf{0}}+\sigma \mathbf{v}_{\mathbf{0}}+\frac{5}{2 \mathcal{R}} \mathbf{v}_{\mathbf{0}}=0
$$

which leads to damped modes with

$$
\operatorname{Re} \sigma=-\frac{5}{2 \mathcal{R}}
$$

Now for $\mathbf{k} \neq 0$ we have solutions with

$$
\begin{gathered}
i \mathbf{k} \cdot \mathbf{v}_{\mathbf{k}}=0, \xi_{\mathbf{k}}=0 \\
\left(\frac{d}{d t}+\sigma+\frac{5}{2 \mathcal{R}}\right)\left(i \mathbf{k} \times \mathbf{v}_{\mathbf{k}}\right)=0
\end{gathered}
$$

leading again to damped modes with $\operatorname{Re} \sigma=-\frac{5}{2 \mathcal{R}}$.

Finally it remains to study the modes such that

$$
i \mathbf{k} \times \mathbf{v}_{\mathbf{k}}=0, i \mathbf{k} \cdot \mathbf{v}_{\mathbf{k}} \neq 0 .
$$

This leads to

$$
\left(\frac{d}{d t}+\sigma+\frac{5}{2 \mathcal{R}}\right)\left(\frac{d}{d t}+\sigma\right) \xi_{\mathbf{k}}+\frac{5 \mathcal{B}}{6} k^{2}\left(f(t)+k^{2}\right) \xi_{\mathbf{k}}=0
$$

where we define $k^{2}=|\mathbf{k}|^{2}$, and where we look for $4 \pi / p$ - periodic solutions $\xi_{\mathrm{k}}$. 
Let us multiply (A.2) by $\overline{\xi_{\mathbf{k}}}$ and integrate over the period $(0,4 \pi)$. Combining real and imaginary parts, integrating by parts once, and writing $\sigma=a+i b$, we obtain

$-\int_{0}^{4 \pi}\left|\dot{\xi}_{\mathbf{k}}\right|^{2} d t+\left(b^{2}+a\left(a+\frac{5}{2 \mathcal{R}}\right)\right) \int_{0}^{4 \pi}\left|\xi_{\mathbf{k}}\right|^{2} d t+\int_{0}^{4 \pi} \frac{5 \mathcal{B}}{6} k^{2}\left(1+\Gamma \cos t+k^{2}\right)\left|\xi_{\mathbf{k}}\right|^{2} d t=0$.

Multiply now (A.2) by $\overline{\dot{\xi}_{\mathrm{k}}}$, and consider the real part, then using the identity

$$
\operatorname{Im} \int_{0}^{4 \pi} \overline{\xi_{\mathbf{k}}} \dot{\xi}_{\mathbf{k}} d t=-b \int_{0}^{4 \pi}\left|\xi_{\mathbf{k}}\right|^{2} d t
$$

we obtain

$$
\left(2 a+\frac{5}{2 \mathcal{R}}\right)\left(\int_{0}^{4 \pi}\left|\dot{\xi}_{\mathbf{k}}\right|^{2} d t-b^{2} \int_{0}^{4 \pi}\left|\xi_{\mathbf{k}}\right|^{2}\right)+\frac{5 \mathcal{B}}{12} \Gamma \int_{0}^{4 \pi} k^{2} \sin t\left|\xi_{\mathbf{k}}\right|^{2} d t=0 .
$$

Hence it results that

$$
\begin{aligned}
& \left(2 a+\frac{5}{2 \mathcal{R}}\right)\left[a\left(a+\frac{5}{2 \mathcal{R}}\right)+\frac{5 \mathcal{B}}{6} k^{2}\left(1+k^{2}\right)\right] \int_{0}^{4 \pi}\left|\xi_{\mathbf{k}}\right|^{2} d t \\
= & -\frac{5 \mathcal{B}}{6} \Gamma k^{2} \int_{0}^{4 \pi}\left(\left(2 a+\frac{5}{2 \mathcal{R}}\right) \cos t+\frac{1}{2} \sin t\right)\left|\xi_{\mathbf{k}}\right|^{2} d t .
\end{aligned}
$$

From the estimate

$$
\left(2 a+\frac{5}{2 \mathcal{R}}\right)\left[a\left(a+\frac{5}{2 \mathcal{R}}\right)+\frac{5 \mathcal{B}}{6} k^{2}\left(1+k^{2}-\Gamma\right)\right] \leq \frac{5 \mathcal{B}}{12} \Gamma k^{2}
$$

we easily deduce that for $\Gamma<1+k^{2}$ and $\mathcal{R}<\frac{5\left(1+k^{2}-\Gamma\right)}{\Gamma}$ then $a<a_{0}(|\mathbf{k}|, \mathcal{B}, \mathcal{R}, \Gamma)<$ 0 . In all other cases $a<a_{0}(|\mathbf{k}|, \mathcal{B}, \mathcal{R}, \Gamma)$ with $a_{0}>0$ (this does not mean that $\sigma$ has a positive real part).

Notice that if we integrate over $(0,4 \pi / p)$ instead of $(0,4 \pi)$, it appears

$$
\frac{5 \mathcal{B}}{12} \Gamma k^{2}\left|\xi_{\mathbf{k}}(0)\right|^{2}(\cos 4 \pi / p-1)
$$

on the left hand side of (A.3). Then using the estimate

$$
\left|\xi_{\mathbf{k}}(0)\right|^{2} \leq(1+p / 4 \pi) \int_{0}^{4 \pi / p}\left|\xi_{\mathbf{k}}\right|^{2} d t+\int_{0}^{4 \pi p}\left|\dot{\xi}_{\mathbf{k}}\right|^{2} d t
$$


we might improve the lower estimates for $\Gamma_{c}(p)$ and $\mathcal{R}_{c}(p)$ as $p \rightarrow \infty$.

From (A.4) it results that for $|\mathbf{k}|$ very large $\operatorname{Re} \sigma=a<0$ (damped mode). Moreover from (A.2) we may proceed with a perturbation analysis for $|\mathbf{k}|$ close to 0 . This leads to consider

$$
\begin{gathered}
\xi_{\mathrm{k}}=1+k^{2} \xi_{1}+O\left(k^{4}\right), \quad \sigma_{\mathbf{k}}=k^{2} \sigma_{1}+O\left(k^{4}\right) \\
\left(\frac{d}{d t}+\frac{5}{2 \mathcal{R}}\right) \frac{d}{d t} \xi_{1}+\frac{5}{2 \mathcal{R}} \sigma_{1}+\frac{5 \mathcal{B}}{6}(1+\Gamma \cos t)=0
\end{gathered}
$$

with $\xi_{1} 4 \pi$ - periodic. This gives indeed

$$
\sigma_{1}=-\frac{\mathcal{B R}}{3}<0
$$

\section{Appendix B. Strategy for computing critical parameter values}

Equation (29) is of Mathieu type

$$
\ddot{u}+\nu \dot{u}+\omega^{2}[1+\gamma \cos t] u=0
$$

with

$$
\nu=\frac{5}{2 \mathcal{R}}, \omega^{2}=\frac{5 \mathcal{B} k^{2}}{6}\left(1+k^{2}\right), \gamma=\frac{\Gamma}{1+k^{2}} .
$$

Using a shooting method, we numerically compute the critical values of $\gamma\left(\nu, \omega^{2}\right)$ for which (B.1) presents a periodic solution. This leads to $\gamma_{0}\left(\nu, \omega^{2}\right)$ and $\gamma_{1}\left(\nu, \omega^{2}\right)$ respectively for $4 \pi$ - periodic and $2 \pi$ - periodic solutions. Now we need to find the minimum of $\Gamma$ with respect to $k^{2}$ :

$$
\Gamma=\left(1+k^{2}\right) \gamma\left(\frac{5}{2 \mathcal{R}}, \frac{5 \mathcal{B} k^{2}}{6}\left(1+k^{2}\right)\right)
$$

This leads for $\gamma_{0}$ and for $\gamma_{1}$, to the conditions

$$
\gamma_{j}+\omega^{2}\left(2+\frac{1}{k^{2}}\right) \frac{\partial \gamma_{j}}{\partial \omega^{2}}=0, j=0
$$

which lead to

$$
\begin{aligned}
k_{c}^{2} & =g_{0}(\mathcal{B}, \mathcal{R}), \\
k_{c}^{\prime 2} & =g_{1}(\mathcal{B}, \mathcal{R}) .
\end{aligned}
$$


Now the fact that $\Gamma_{c}$ is the same for $4 \pi$ - periodic and for $2 \pi$ - periodic solutions, gives the identity

$$
\left(1+k_{c}^{2}\right) \gamma_{0}\left(\frac{5}{2 \mathcal{R}}, \frac{5 \mathcal{B}}{6} k_{c}^{2}\left(1+k_{c}^{2}\right)\right)=\left(1+k_{c}^{\prime 2}\right) \gamma_{1}\left(\frac{5}{2 \mathcal{R}}, \frac{5 \mathcal{B}}{6} k_{c}^{\prime 2}\left(1+k_{c}^{\prime 2}\right)\right)
$$

which, by using (B.2) leads to a relation of the form

$$
h(\mathcal{B}, \mathcal{R})=0 .
$$

The relation (16) then gives

$$
\cos ^{2} \frac{n \pi}{q}=\frac{g_{1}(\mathcal{B}, \mathcal{R})}{4 g_{0}(\mathcal{B}, \mathcal{R})}
$$

while relation (18) gives

$$
\cos ^{2} \frac{(2 n-1) \pi}{2 q}=\frac{g_{1}(\mathcal{B}, \mathcal{R})}{4 g_{0}(\mathcal{B}, \mathcal{R})} .
$$

Hence, for a ratio $n / q$ or $(2 n-1) / 2 q$ fixed, we find a second relation between $\mathcal{R}$ and $\mathcal{B}$.

In order to compute numerical values for the coefficients of the normal forms, we first solve numerically (29) with a Runge-Kutta method, precise at fourth order. The time step of integration is $10^{-3}$. Using a shooting method, we obtain, for a given set of parameters $(\mathcal{R}, \mathcal{B})$, the values of $(\Gamma, k)$ such that $4 \pi$ and $2 \pi$ periodic functions become solutions of (29). Since we are interested for the minimal values of the forcing term $\Gamma$, we numerically locate $k_{c}$ and $k_{c}^{\prime}$ that render minimal $\Gamma_{c}(0, \mathcal{R}, \mathcal{B})$ and $\Gamma_{c}(1, \mathcal{R}, \mathcal{B})$. Using a Newton algorithm, we numerically compute a set $\left(\mathcal{R}_{c}, \mathcal{B}_{c}\right)$ for a given $(n, q)$, for which the two minima $\Gamma_{c}(0, \mathcal{R}, \mathcal{B})$ and $\Gamma_{c}(1, \mathcal{R}, \mathcal{B})$ becomes equal. This method is equivalent to find the roots of the equations (B.3,B.4). In Figure B.4 we present the marginal curves for which $n=1, q=5$, i.e. $\cos (\pi / 5)=k_{c}^{\prime} / 2 k_{c}$ and $n=1, q=7$, i.e. $\cos (\pi / 7)=k_{c}^{\prime} / 2 k_{c}$.

\section{Appendix C. Amplitude equations for $q$ even}

When $q$ is even in Hypothesis $2 \mathrm{a}$ or $2 \mathrm{~b}$, we need to satisfy the following symmetry properties (see (23)):

$$
\begin{aligned}
\boldsymbol{\Psi}\left(A_{0}, A_{1}, B_{0}, B_{1}, \mu, \beta, \gamma, t+2 \pi\right) & =\boldsymbol{\Psi}\left(-A_{0}, A_{1},-B_{0}, B_{1}, \mu, \beta, \gamma, t\right), \\
\mathbf{R}_{\pi / q} \boldsymbol{\Psi}\left(A_{0}, A_{1}, B_{0}, B_{1}, \mu, \beta, \gamma, t\right) & =\boldsymbol{\Psi}\left(B_{0}, B_{1}, A_{0}, A_{1}, \mu, \beta, \gamma, t\right),
\end{aligned}
$$



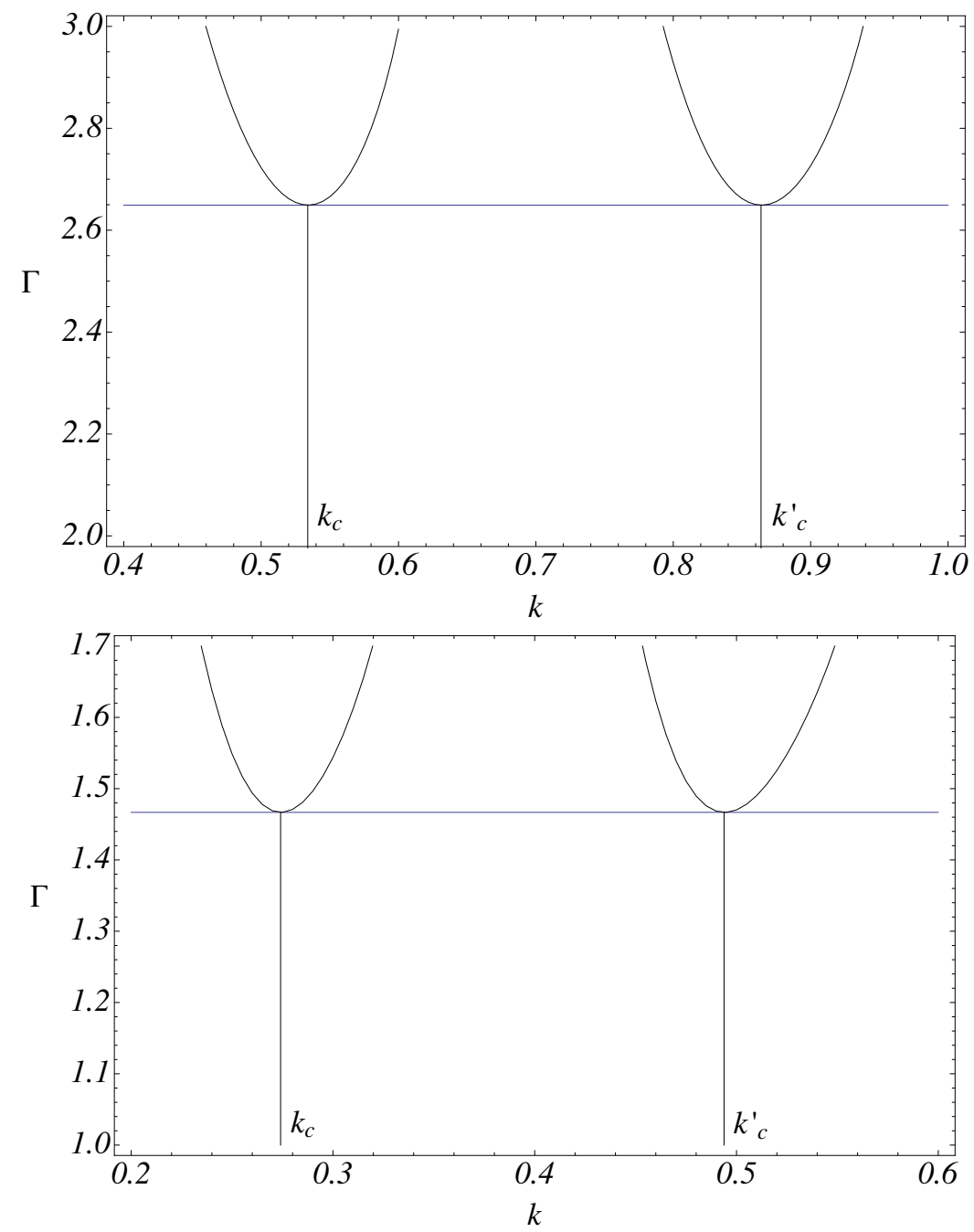

Figure B.4: Neutral stability curves. top: the parameter values are $\left(\mathcal{B}_{c}, \mathcal{R}_{c}\right)=(1.27,4.07)$, for which $n=1$, and $q=5, k_{c}^{\prime} / k_{c}=2 \cos \pi / 5$. Bottom: $\left(\mathcal{B}_{c}, \mathcal{R}_{c}\right)=(5.10,6.47)$, for which $n=1$ and $q=7, k_{c}^{\prime} / k_{c}=2 \cos \pi / 7$. 
and the system of amplitude equations satisfied by $\left(A_{0}, A_{1}, \overline{A_{0}}, \overline{A_{1}}\right)$ needs to commute with the actions

$$
\begin{aligned}
& \left(A_{0}, A_{1}, B_{0}, B_{1}, t\right) \rightarrow\left(-A_{0}, A_{1},-B_{0}, B_{1}, t\right), \\
& \left(A_{0}, A_{1}, B_{0}, B_{1}, t\right) \rightarrow\left(B_{0}, B_{1}, A_{0}, A_{1}, t\right),
\end{aligned}
$$

representing respectively the time shift $t \rightarrow t+2 \pi$ and the rotation $R_{\pi / q}$, and, because of the non trivial action of $\mathbf{S}$ in case of Hypothesis $2 \mathrm{~b}$ :

$$
\left(A_{0}, A_{1}, B_{0}, B_{1}, t\right) \rightarrow\left(A_{0}, B_{1}, B_{0}, A_{1}, t\right) .
$$

All eigenvectors being real, it results that the coefficients of the amplitude equations are real, and the time shift $t \rightarrow t+2 \pi$ invariance leads to the component which corresponds to $d A_{0} / d t$ odd in $A_{0}, B_{0}$, and the component $d A_{1} / d t$ even in $A_{0}, B_{0}$.

Moreover, as above, we need to keep in mind that in the normal form the component $d A_{0} / d t$ results from a scalar product with $\boldsymbol{\zeta}_{0}^{*}(t)$, i.e. with Fourier components of the form $e^{i \mathbf{k}_{2 j-1} \cdot \mathbf{x}}$ with $1 \leq j \leq q$, while the component $d A_{1} / d t$ results from a scalar product with $\boldsymbol{\zeta}_{1}^{*}(t)$, i.e. with Fourier components of the form $e^{i \mathbf{k}_{2 j-1}^{\prime} \cdot \mathbf{x}}$ with $1 \leq j \leq q$ for Hypothesis 2 a, or of the form $e^{i \mathbf{k}_{2 j-1 / 2}^{\prime} \cdot \mathbf{x}}$ with $1 \leq j \leq q$ for Hypothesis 2b. By construction we have

i) for $q$ even and Hypothesis $2 \mathrm{a}$

$$
\mathbf{k}_{j}+\mathbf{k}_{j+2 n}=\mathbf{k}_{j+n}^{\prime}
$$

and quadratic resonances occur when $\mathbf{k}_{1}=\mathbf{k}_{1 \pm n}^{\prime}+\mathbf{k}_{1 \pm 2 n+q}$ for $\frac{d A_{0}}{d t}$, and $\mathbf{k}_{1}^{\prime}=\mathbf{k}_{1-n}+\mathbf{k}_{1+n}$ for $\frac{d A_{1}}{d t}$. The form of the system of amplitude equations then depends again on the parity of $n$. We obtain at cubic order for Hypothesis $2 a$ with $q$ even:

For $n$ odd

$$
\begin{aligned}
\frac{d A_{0}}{d t} & =\mu_{0} A_{0}+a_{0} A_{0} B_{1}+b_{0} A_{0}^{3}+c_{0} A_{0} B_{0}^{2}+d_{0} A_{0} A_{1}^{2}+e_{0} A_{0} B_{1}^{2} \\
\frac{d B_{0}}{d t} & =\mu_{0} B_{0}+a_{0} B_{0} A_{1}+b_{0} B_{0}^{3}+c_{0} B_{0} A_{0}^{2}+d_{0} B_{0} B_{1}^{2}+e_{0} B_{0} A_{1}^{2} \\
\frac{d A_{1}}{d t} & =\mu_{1} A_{1}+a_{1} B_{0}^{2}+b_{1} A_{1}^{3}+c_{1} A_{1} B_{1}^{2}+d_{1} A_{1} A_{0}^{2}+e_{1} A_{1} B_{0}^{2} \\
\frac{d B_{1}}{d t} & =\mu_{1} B_{1}+a_{1} A_{0}^{2}+b_{1} B_{1}^{3}+c_{1} B_{1} A_{1}^{2}+d_{1} B_{1} B_{0}^{2}+e_{1} B_{1} A_{0}^{2}
\end{aligned}
$$


For $n$ even

$$
\begin{aligned}
\frac{d A_{0}}{d t} & =\mu_{0} A_{0}+a_{0} A_{0} A_{1}+b_{0} A_{0}^{3}+c_{0} A_{0} B_{0}^{2}+d_{0} A_{0} A_{1}^{2}+e_{0} A_{0} B_{1}^{2}, \\
\frac{d B_{0}}{d t} & =\mu_{0} B_{0}+a_{0} B_{0} B_{1}+b_{0} B_{0}^{3}+c_{0} B_{0} A_{0}^{2}+d_{0} B_{0} B_{1}^{2}+e_{0} B_{0} A_{1}^{2}, \\
\frac{d A_{1}}{d t} & =\mu_{1} A_{1}+a_{1} A_{0}^{2}+b_{1} A_{1}^{3}+c_{1} A_{1} B_{1}^{2}+d_{1} A_{1} A_{0}^{2}+e_{1} A_{1} B_{0}^{2}, \\
\frac{d B_{1}}{d t} & =\mu_{1} B_{1}+a_{1} B_{0}^{2}+a_{1} A_{0}^{2}+b_{1} B_{1}^{3}+c_{1} B_{1} A_{1}^{2}+d_{1} B_{1} B_{0}^{2}+e_{1} B_{1} A_{0}^{2} .
\end{aligned}
$$

ii) For $q$ even and Hypothesis $2 \mathrm{~b}$

$$
\mathbf{k}_{j}+\mathbf{k}_{j+2 n-1}=\mathbf{k}_{j+n-1 / 2}^{\prime}
$$

and quadratic resonances occur when $\mathbf{k}_{1}=\mathbf{k}_{n+1 / 2}^{\prime}+\mathbf{k}_{2 n+q}$ and $\mathbf{k}_{1}=\mathbf{k}_{1-n+1 / 2}^{\prime}+$ $\mathbf{k}_{2-2 n+q}$ for $\frac{d A_{0}}{d t}$, and when $\mathbf{k}_{1+1 / 2}^{\prime}=\mathbf{k}_{2-n}+\mathbf{k}_{2+n-1}$ for $\frac{d A_{1}}{d t}$, which leads to (using the equivariance under $\mathbf{S}$ )

$$
\begin{aligned}
\frac{d A_{0}}{d t} & =\mu_{0} A_{0}+a_{0} A_{0}\left(A_{1}+B_{1}\right)+b_{0} A_{0}^{3}+c_{0} A_{0} B_{0}^{2}+d_{0} A_{0}\left(A_{1}^{2}+B_{1}^{2}\right) \\
\frac{d B_{0}}{d t} & =\mu_{0} B_{0}+a_{0} B_{0}\left(A_{1}+B_{1}\right)+b_{0} B_{0}^{3}+c_{0} B_{0} A_{0}^{2}+d_{0} B_{0}\left(A_{1}^{2}+B_{1}^{2}\right)(, \\
\frac{d A_{1}}{d t} & =\mu_{1} A_{1}+a_{1} A_{0} B_{0}+b_{1} A_{1}^{3}+c_{1} A_{1} B_{1}^{2}+d_{1} A_{1}\left(A_{0}^{2}+B_{0}^{2}\right) \\
\frac{d B_{1}}{d t} & =\mu_{1} B_{1}+a_{1} A_{0} B_{0}+b_{1} B_{1}^{3}+c_{1} B_{1} A_{1}^{2}+d_{1} B_{1}\left(A_{0}^{2}+B_{0}^{2}\right)
\end{aligned}
$$

\section{Appendix D. Equilibrium solutions and their stability in the case $q$ even}

Appendix D.1. Monomodal solutions of (C.2), (C.3) and (C.4)

Non trivial monomodal solutions are given by

$$
\begin{aligned}
A_{0}=B_{0} & =0 \\
A_{1}\left(\mu_{1}+b_{1} A_{1}^{2}+c_{1} B_{1}^{2}\right) & =0 \\
B_{1}\left(\mu_{1}+b_{1} B_{1}^{2}+c_{1} A_{1}^{2}\right) & =0
\end{aligned}
$$

which corresponds to solutions built on the lattice spanned only with wave vectors $\mathbf{k}_{j}^{\prime}$ or $\mathbf{k}_{j+1 / 2}^{\prime}$. 
i) For $A_{1}=0$, the solutions are invariant under $\mathbf{R}_{2 \pi / q}$ and exchanged by $\mathbf{R}_{\pi / q}$ into the solutions with $B_{1}=0$. Necessary conditions for the stability of these solutions are (for the two solutions $A_{1}=0, B_{1}= \pm \sqrt{\mu_{1} /-b_{1}}$ )

$$
\mu_{1}>0, \quad b_{1}<0, \quad \mu_{0}-d_{0} \mu_{1} / b_{1}<0, \quad \mu_{0}-e_{0} \mu_{1} / b_{1}+a_{0} B_{1}<0 .
$$

ii) For $A_{1} B_{1} \neq 0$, and if $b_{1} \neq c_{1}$ we have

$$
A_{1}^{2}=B_{1}^{2}=-\frac{\mu_{1}}{b_{1}+c_{1}}
$$

which gives 4 solutions. For $A_{1}=B_{1}$ (two cases) they are invariant under $\mathbf{R}_{\pi / q}$, while for $A_{1}=-B_{1}$ (two cases) they are exchanged by $\mathbf{R}_{\pi / q}$. Necessary conditions for the stability of these solutions are

$$
\begin{aligned}
\mu_{1} & >0, \\
b_{1}+\left|c_{1}\right| & <0, \\
\mu_{0}-\frac{\left(d_{0}+e_{0}\right)}{b_{1}+c_{1}} \mu_{1}+\max \left\{a_{0} B_{1}, a_{0} A_{1}\right\} & <0,
\end{aligned}
$$

Appendix D.2. Bimodal equilibrium solutions for $q$ even

i) For $n$ odd, $B_{0}=A_{1}=0, A_{0} \neq 0$ we have from (C.2)

$$
\begin{aligned}
\mu_{0}+a_{0} B_{1}+b_{0} A_{0}^{2}+e_{0} B_{1}^{2} & =0, \\
\mu_{1} B_{1}+a_{1} A_{0}^{2}+b_{1} B_{1}^{3}+e_{1} B_{1} A_{0}^{2} & =0,
\end{aligned}
$$

hence we obtain two solutions (depending on the sign of $A_{0}$ ) with

$$
B_{1}=-\frac{\mu_{0}}{a_{0}}+\text { h.o.t., } \quad A_{0}^{2}=\frac{\mu_{0} \mu_{1}}{a_{0} a_{1}}+\text { h.o.t. }
$$

The time shift by $2 \pi$ exchanges the two solutions. A necessary stability condition for these solutions is that the 4 eigenvalues of the linearized system (representing the principal part of Floquet exponents for the full system) have a negative real part, which gives (at main order)

$$
\mu_{0}<0, \mu_{1}>0, a_{0} a_{1}<0, a_{0}^{2} \mu_{1}+c_{1} \mu_{0}^{2}<0, \frac{a_{0} b_{0}}{a_{1}} \mu_{1}+b_{1} \mu_{0}<0 .
$$

ii) Still for $n$ odd, $A_{0}=B_{1}=0, B_{0} \neq 0$ represents the solutions deduced from the previous ones by the rotation $\mathbf{R}_{\pi / q}$. The stability conditions are the same as above. 
iii) Still for $n$ odd, $B_{0}=0, A_{0} A_{1} \neq 0$. Equilibria should satisfy the system

$$
\begin{aligned}
\mu_{0}+a_{0} B_{1}+b_{0} A_{0}^{2}+d_{0} A_{1}^{2}+e_{0} B_{1}^{2} & =0, \\
\mu_{1}+b_{1} A^{2}+c_{1} B_{1}^{2}+d_{1} A_{0}^{2} & =0 \\
\mu_{1} B_{1}+a_{1} A_{0}^{2}+b_{1} B_{1}^{3}+c_{1} B_{1} A_{1}^{2}+e_{1} B_{1} A_{0}^{2} & =0,
\end{aligned}
$$

which leads to 4 solutions given by

$$
\begin{aligned}
& B_{1}=\frac{1}{a_{0} b_{1}}\left(d_{0} \mu_{1}-b_{1} \mu_{0}\right)+\text { h.o.t. } \\
& A_{1}^{2}=-\frac{\mu_{1}}{b_{1}}+\text { h.o.t. } \\
& A_{0}^{2}=\frac{\left(c_{1}-b_{1}\right)}{a_{0} a_{1} b_{1}^{2}} \mu_{1}\left(d_{0} \mu_{1}-b_{1} \mu_{0}\right)+\text { h.o.t. }
\end{aligned}
$$

The time shift of $2 \pi$ exchanges two solutions with opposite $A_{0}$, all these solutions being $4 \pi$ - time periodic and invariant under $\mathbf{R}_{2 \pi / q}$. The action of $\mathbf{R}_{\pi / q}$ leads to the other family of solutions with $A_{0}=0, B_{0} B_{1} \neq 0$.

The study of the 4 eigenvalues of the linearized system (C.2) around these solutions, leads (for the principal parts) to the characteristic polynomial

$$
\left(\lambda-\mu_{0}-a_{0} A_{1}\right)\left(\lambda^{3}+\frac{\left(c_{1}-b_{1}\right)}{b_{1}} \mu_{1} \lambda^{2}+\frac{2\left(c_{1}-b_{1}\right)}{b_{1}} \mu_{1}\left[\left(1-\frac{d_{0}}{b_{1}}\right) \mu_{1}+\mu_{0}\right] \lambda+4 a_{0} a_{1} \mu_{1} A_{0}^{2}\right)=0 .
$$

Using the Routh-Hurwitz criterium, we obtain the following necessary stability conditions (combined with existence conditions)

$$
\begin{aligned}
\frac{\mu_{1}}{b_{1}} & <0, c_{1}-b_{1}<0, d_{0} \mu_{1}-b_{1} \mu_{0}<0, \mu_{0}+a_{0} A_{1}<0, \\
a_{0} a_{1} \mu_{1} & >0,2\left(d_{0} \mu_{1}-b_{1} \mu_{0}\right)+\left(b_{1}-c_{1}\right)\left[\left(1-\frac{d_{0}}{b_{1}}\right) \mu_{1}+\mu_{0}\right]>0 .
\end{aligned}
$$

As above, this gives the negativity of the real part of Floquet exponents for the full system, but the stability is not proved because of the small divisor problem mentioned above, giving not isolated eigenvalues.

iv) Still for $n$ odd, $A_{0} B_{0} \neq 0$. Equilibria are solution of

$$
\begin{aligned}
\mu_{0}+a_{0} B_{1}+b_{0} A_{0}^{2}+c_{0} B_{0}^{2}+d_{0} A_{1}^{2}+e_{0} B_{1}^{2} & =0 \\
\mu_{0}+a_{0} A_{1}+b_{0} B_{0}^{2}+c_{0} A_{0}^{2}+d_{0} B_{1}^{2}+e_{0} A_{1}^{2} & =0 \\
\mu_{1} A_{1}+a_{1} B_{0}^{2}+b_{1} A_{1}^{3}+c_{1} A_{1} B_{1}^{2}+d_{1} A_{1} A_{0}^{2}+e_{1} A_{1} B_{0}^{2} & =0 \\
\mu_{1} B_{1}+a_{1} A_{0}^{2}+b_{1} B_{1}^{3}+c_{1} B_{1} A_{1}^{2}+d_{1} B_{1} B_{0}^{2}+e_{1} B_{1} A_{0}^{2} & =0 .
\end{aligned}
$$


Combining the equations above we obtain

$$
\begin{aligned}
0= & \left(B_{1}-A_{1}\right)\left[a_{0}+\left(e_{0}-d_{0}\right)\left(A_{1}+B_{1}\right)\right]+\left(b_{0}-c_{0}\right)\left(A_{0}^{2}-B_{0}^{2}\right), \\
0= & \left(B_{1}-A_{1}\right)\left[\mu_{1}+b_{1}\left(A_{1}^{2}+A_{1} B_{1}+B_{1}^{2}\right)-c_{1} A_{1} B_{1}+d_{1} B_{0}^{2}+e_{1} A_{0}^{2}\right]+ \\
& +\left(A_{0}^{2}-B_{0}^{2}\right)\left[a_{1}-d_{1} A_{1}-e_{1} A_{1}\right]
\end{aligned}
$$

which generically (if $a_{0} a_{1} \neq 0$ ) leads uniquely to (for solutions close to 0 )

$$
A_{1}=B_{1}, A_{0}^{2}=B_{0}^{2} .
$$

Now, we just need to solve

$$
\begin{aligned}
\mu_{0}+a_{0} A_{1}+\left(b_{0}+c_{0}\right) A_{0}^{2}+\left(d_{0}+e_{0}\right) A_{1}^{2} & =0 \\
\mu_{1} A_{1}+a_{1} A_{0}^{2}+\left(b_{1}+c_{1}\right) A_{1}^{3}+\left(d_{1}+e_{1}\right) A_{1} A_{0}^{2} & =0
\end{aligned}
$$

which leads to

$$
\begin{aligned}
& B_{0}^{2}=A_{0}^{2}=\frac{\mu_{0} \mu_{1}}{a_{0} a_{1}}+\text { h.o.t. } \\
& A_{1}=B_{1}=-\frac{\mu_{0}}{a_{0}}+\text { h.o.t.. }
\end{aligned}
$$

These are four solutions invariant or exchanged by $\mathbf{R}_{\pi / q}$.

The study of the 4 eigenvalues of the linearized system (C.2) around these solutions, leads (for the principal parts) to the characteristic polynomial

$$
\lambda^{4}-2 \mu_{1} \lambda^{3}+\left(\mu_{1}^{2}-4 \mu_{0} \mu_{1}\right) \lambda^{2}+4 \mu_{0} \mu_{1}^{2} \lambda+4 \mu_{0}^{2} \mu_{1}^{2}=0 .
$$

Using the Routh-Hurwitz criterium, we obtain the following necessary stability conditions (combined with existence conditions)

$$
\mu_{1}<0, \quad \mu_{0}>0, \quad a_{0} a_{1}<0 .
$$

As above, this gives the negativity of the real part of Floquet exponents for the full system, but the stability is not proved because of the small divisor problem mentioned above, giving not isolated eigenvalues.

v) For $q$ even and $n$ even, the set of equilibria is easily obtained in changing $A_{1}$ in $B_{1}$ in the previous study made for $n$ odd.

vi) For $q$ even and Hypothesis $2 \mathrm{~b}$ (see system (C.4)), $B_{0}=A_{1}=0$, $A_{0} \neq 0$, equilibria need to solve

$$
\begin{aligned}
\mu_{0}+a_{0} B_{1}+b_{0} A_{0}^{2}+d_{0} B_{1}^{2} & =0 \\
\mu_{1}+b_{1} B_{1}^{2}+d_{1} A_{0}^{2} & =0
\end{aligned}
$$


which gives

$$
\begin{aligned}
& B_{1}=\frac{1}{a_{0} d_{1}}\left(b_{0} \mu_{1}-d_{1} \mu_{0}\right)+\text { h.o.t. } \\
& A_{0}^{2}=-\frac{\mu_{1}}{d_{1}}+\text { h.o.t. }
\end{aligned}
$$

These two solutions may exist only if at main order, $\mu_{1} d_{1}<0$. The characteristic polynomial giving the principal part of eigenvalues for the stability study is as follows:

$$
\left(\lambda+\left(b_{0}-c_{0}\right) A_{0}^{2}\right)\left(\lambda^{3}+\frac{2 b_{0} \mu_{1}}{d_{1}} \lambda^{2}+\frac{2 \mu_{1}}{d_{1}}\left(b_{0} \mu_{1}-d_{1} \mu_{0}\right) \lambda+\frac{2\left(b_{1}-c_{1}\right) \mu_{1}}{a_{0}^{2} d_{1}^{3}}\left(b_{0} \mu_{1}-d_{1} \mu_{0}\right)^{3}=0,\right.
$$

which, by using the Routh - Hurwitz criterium combined with the existence condition, leads to necessary conditions (at main order) for the stability of these solutions:

$$
\mu_{1} d_{1}<0, b_{0}<0, b_{1}-c_{1}>0, b_{0}-c_{0}>0, b_{0} \mu_{1}-d_{1} \mu_{0}<0 .
$$

via) For $q$ even, Hypothesis $2 \mathrm{~b}, B_{0}=B_{1}=0, A_{0} \neq 0$, equilibria are deduced from the previous one by using the symmetry $\mathbf{S}$.

vii) For $q$ even, Hypothesis $2 \mathrm{~b}, B_{0}=0, A_{0} A_{1} B_{1} \neq 0$, equilibria need to solve

$$
\begin{aligned}
\mu_{0}+a_{0}\left(A_{1}+B_{1}\right)+b_{0} A_{0}^{2}+d_{0}\left(A_{1}^{2}+B_{1}^{2}\right) & =0 \\
\mu_{1}+b_{1} A_{1}^{2}+c_{1} B_{1}^{2}+d_{1} A_{0}^{2} & =0 \\
\mu_{1}+b_{1} B_{1}^{2}+c_{1} A_{1}^{2}+d_{1} A_{0}^{2} & =0 .
\end{aligned}
$$

It is clear that if $b_{1} \neq c_{1}$, this leads to two different cases: either $A_{1}=B_{1}$, or $A_{1}=-B_{1}$.

viia) For $q$ even, Hypothesis $2 \mathrm{~b}, A_{1}=B_{1}, B_{0}=0$. This gives

$$
\begin{aligned}
\mu_{0}+2 a_{0} A_{1}+b_{0} A_{0}^{2}+2 d_{0} A_{1}^{2} & =0 \\
\mu_{1}+\left(b_{1}+c_{1}\right) A_{1}^{2}+d_{1} A_{0}^{2} & =0
\end{aligned}
$$

hence

$$
\begin{aligned}
& A_{1}=B_{1}=\frac{d_{1} \mu_{0}-b_{0} \mu_{1}}{\left(b_{1}+c_{1}\right) b_{0}-2 a_{0} d_{1}}+\text { h.o.t. } \\
& A_{0}^{2}=\frac{2 a_{0} \mu_{1}-\left(b_{1}+c_{1}\right) \mu_{0}}{\left(b_{1}+c_{1}\right) b_{0}-2 a_{0} d_{1}}+\text { h.o.t. }
\end{aligned}
$$


These two solutions are invariant under symmetry $\mathbf{S}$, and exchanged into one another by the $2 \pi$ time shift. The characteristic polynomial is here given at main order by

$$
\left[\left(c_{0}-b_{0}\right) A_{0}^{2}-\lambda\right]\left[-\lambda^{3}+2 b_{0} A_{0}^{2} \lambda^{2}+4 a_{0} d_{1} A_{0}^{2} A_{1} \lambda+8 a_{0} d_{1}\left(c_{1}-b_{1}\right) A_{0}^{2} A_{1}^{3}\right]=0 .
$$

This leads to necessary conditions for existence and stability of these solutions (at main order):

$$
\frac{2 a_{0} \mu_{1}-\left(b_{1}+c_{1}\right) \mu_{0}}{\left(b_{1}+c_{1}\right) b_{0}-2 a_{0} d_{1}}>0, b_{0}<0, c_{0}-b_{0}<0, b_{1}-c_{1}<0, a_{0} d_{1} \frac{d_{1} \mu_{0}-b_{0} \mu_{1}}{\left(b_{1}+c_{1}\right) b_{0}-2 a_{0} d_{1}}<0 .
$$

viib) For $q$ even, Hypothesis $2 \mathrm{~b}, A_{1}=-B_{1}, B_{0}=0$. This gives

$$
\begin{aligned}
\mu_{0}+b_{0} A_{0}^{2}+2 d_{0} A_{1}^{2} & =0 \\
\mu_{1}+\left(b_{1}+c_{1}\right) A_{1}^{2}+d_{1} A_{0}^{2} & =0
\end{aligned}
$$

hence

$$
\begin{aligned}
A_{0}^{2} & =\frac{2 d_{0} \mu_{1}-\left(b_{1}+c_{1}\right) \mu_{0}}{\left(b_{1}+c_{1}\right) b_{0}-2 d_{0} d_{1}}, \\
A_{1}^{2} & =\frac{d_{1} \mu_{0}-b_{0} \mu_{1}}{\left(b_{1}+c_{1}\right) b_{0}-2 d_{0} d_{1}} .
\end{aligned}
$$

This corresponds to four solutions transformed into each other by using combinations of the $2 \pi$ time shift and the symmetry $\mathbf{S}$. The characteristic polynomial is here given at main order by

$$
\left[\left(c_{0}-b_{0}\right) A_{0}^{2}-\lambda\right]\left(2 b_{0} A_{0}^{2}-\lambda\right)\left(2 b_{1} A_{1}^{2}-\lambda\right)^{2}=0,
$$

which leads to the following necessary existence and stability conditions

$$
\frac{2 d_{0} \mu_{1}-\left(b_{1}+c_{1}\right) \mu_{0}}{\left(b_{1}+c_{1}\right) b_{0}-2 d_{0} d_{1}}>0, \frac{d_{1} \mu_{0}-b_{0} \mu_{1}}{\left(b_{1}+c_{1}\right) b_{0}-2 d_{0} d_{1}}>0, c_{0}-b_{0}<0, b_{0}<0, b_{1}<0 \text {. }
$$

viii) For $q$ even, Hypothesis $2 \mathrm{~b}, A_{0}=0, B_{0} \neq 0$. Equilibria are deduced from the ones in cases vi), via), viia), viib) by using rotation $\mathbf{R}_{\pi / q}$.

ix) For $q$ even, Hypothesis $2 b, A_{0} B_{0} \neq 0$. Equilibria should satisfy

$$
\begin{aligned}
\mu_{0}+a_{0}\left(A_{1}+B_{1}\right)+b_{0} A_{0}^{2}+c_{0} B_{0}^{2}+d_{0}\left(A_{1}^{2}+B_{1}^{2}\right) & =0 \\
\mu_{0}+a_{0}\left(A_{1}+B_{1}\right)+b_{0} B_{0}^{2}+c_{0} A_{0}^{2}+d_{0}\left(A_{1}^{2}+B_{1}^{2}\right) & =0 \\
\mu_{1} A_{1}+a_{1} A_{0} B_{0}+b_{1} A_{1}^{3}+c_{1} A_{1} B_{1}^{2}+d_{1} A_{1}\left(A_{0}^{2}+B_{0}^{2}\right) & =0 \\
\mu_{1} B_{1}+a_{1} A_{0} B_{0}+b_{1} B_{1}^{3}+c_{1} B_{1} A_{1}^{2}+d_{1} B_{1}\left(A_{0}^{2}+B_{0}^{2}\right) & =0,
\end{aligned}
$$


and it is easy to see that if $b_{0}-c_{0} \neq 0$, then

$$
\begin{aligned}
A_{0} & = \pm B_{0}, \\
0 & =\left(A_{1}-B_{1}\right)\left[\mu_{1}-\left(b_{1}+c_{1}\right) A_{1} B_{1}+2 d_{1} A_{0}^{2}+b_{1}\left(A_{1}+B_{1}\right)^{2}\right],
\end{aligned}
$$

ixa) For $A_{1}=B_{1}, A_{0}= \pm B_{0}$, this gives

$$
\begin{aligned}
\mu_{0}+2 a_{0} A_{1}+\left(b_{0}+c_{0}\right) A_{0}^{2}+2 d_{0} A_{1}^{2} & =0, \\
\mu_{1} A_{1} \pm a_{1} A_{0}^{2}+\left(b_{1}+c_{1}\right) A_{1}^{3}+2 d_{1} A_{1} A_{0}^{2} & =0,
\end{aligned}
$$

hence

$$
\begin{aligned}
& A_{1}=B_{1}=-\frac{\mu_{0}}{2 a_{0}}+\text { h.o.t. } \\
& A_{0}^{2}=B_{0}^{2}= \pm \frac{\mu_{0} \mu_{1}}{2 a_{0} a_{1}}+\text { h.o.t. }
\end{aligned}
$$

This corresponds to two solutions (only one sign + or - is possible in $A_{0}^{2}$ ) invariant under symmetry $\mathbf{S}$, either invariant under the rotation $\mathbf{R}_{\pi / q}\left(A_{0}=\right.$ $B_{0}$ case), or exchanged into one another by $\mathbf{R}_{\pi / q}\left(A_{0}=-B_{0}\right)$. The $2 \pi$ time shift transforms one solution into the other in all cases. The characteristic polynomial is here given at main order by

$$
\left[2\left(b_{0}-c_{0}\right) A_{0}^{2}-\lambda\right]\left(\mu_{1}-\lambda\right)\left[\lambda^{2}-\mu_{1} \lambda \mp 4 a_{0} a_{1} A_{0}^{2}\right]=0,
$$

leading at main order to the necessary stability conditions

$$
\begin{aligned}
\mu_{1} & <0, b_{0}-c_{0}<0 \\
a_{0} a_{1} & <0 \text { for } A_{0}=B_{0}, a_{0} a_{1}>0 \text { for } A_{0}=-B_{0} .
\end{aligned}
$$

ixb) For $A_{1}-B_{1} \neq 0, A_{0}= \pm B_{0}$, equilibria should satisfy

$$
\begin{aligned}
\mu_{0}+a_{0}\left(A_{1}+B_{1}\right)+\left(b_{0}+c_{0}\right) A_{0}^{2}-2 d_{0} A_{1} B_{1}+d_{0}\left(A_{1}+B_{1}\right)^{2} & =0 \\
\mu_{1}-\left(b_{1}+c_{1}\right) A_{1} B_{1}+2 d_{1} A_{0}^{2}+b_{1}\left(A_{1}+B_{1}\right)^{2} & =0 \\
\pm a_{1} A_{0}^{2}+\left(c_{1}-b_{1}\right)\left(A_{1}+B_{1}\right) A_{1} B_{1} & =0
\end{aligned}
$$

hence

$$
\begin{aligned}
A_{1}+B_{1} & =\frac{2 d_{0} \mu_{1}-\left(b_{1}+c_{1}\right) \mu_{0}}{a_{0}\left(b_{1}+c_{1}\right)}+\text { h.o.t. } \\
A_{1} B_{1} & =\frac{\mu_{1}}{b_{1}+c_{1}}+\text { h.o.t., } \\
A_{0}^{2} & =B_{0}^{2}= \pm \frac{\mu_{1}\left(b_{1}-c_{1}\right)\left(2 d_{0} \mu_{1}-\left(b_{1}+c_{1}\right) \mu_{0}\right)}{a_{0} a_{1}\left(b_{1}+c_{1}\right)^{2}}+\text { h.o.t. }
\end{aligned}
$$


This corresponds to eight solutions ( 4 with $A_{0}=B_{0}, 4$ with $A_{0}=-B_{0}$ ), the existence condition being at main order

$$
\mu_{1}\left(b_{1}+c_{1}\right)<0
$$

In each family, the four solutions are deduced from one of them in playing with $\mathbf{R}_{\pi / q}, \mathbf{S}$, and the $2 \pi$ time shift. The characteristic polynomial is here given at main order by

$$
\left[2\left(b_{0}-c_{0}\right) A_{0}^{2}-\lambda\right]\left[-\lambda^{3}-4 \mu_{1} \lambda^{2}-4 \mu_{1}^{2} \frac{b_{1}-c_{1}}{b_{1}+c_{1}} \lambda \pm 8 a_{0} a_{1} \mu_{1} A_{0}^{2}\right]=0,
$$

leading at main order, to the necessary stability conditions

$$
\begin{aligned}
\mu_{1} & >0, b_{0}-c_{0}<0, b_{1}+c_{1}<0, b_{1}-c_{1}<0, \\
a_{0} a_{1} & <0 \text { for } A_{0}=B_{0}, a_{0} a_{1}>0 \text { for } A_{0}=-B_{0}, \\
2 \mu_{1}^{2}\left(b_{1}^{2}-c_{1}^{2}\right) & >\left|a_{0} a_{1}\right|\left(b_{1}+c_{1}\right)^{2} A_{0}^{2} .
\end{aligned}
$$

\section{Appendix E. Computation of coefficients}

Let us compute quadratic coefficients $a_{0}, a_{1}$ in (36), (37), (38), (C.2), (C.3), (C.4).

We start with (32), where $\boldsymbol{\Psi}$ satisfies (33) and we make $\mu=\beta=\gamma=0$. Then the Taylor expansion of $\boldsymbol{\Psi}$ takes the form

$$
\boldsymbol{\Psi}\left(A_{0}, B_{0}, A_{1}, B_{1}\right)=\sum_{p+q+r+s \geq 2} A_{0}^{p} B_{0}^{q} A_{1}^{r} B_{1}^{s} \boldsymbol{\Psi}_{\text {pqrs }}
$$

and then write $d U / d t$ in two different ways: i) by using the time derivative of (32) with (36) for example with $B_{j}=\overline{A_{j}}$, ii) by using (4) with (32). We identify powers of $A_{0}, B_{0}, A_{1}, B_{1}$ at successive orders and obtain for system (36):

$$
\begin{aligned}
& a_{0} \boldsymbol{\zeta}_{0}+\left(\frac{d}{d t}-\mathbf{L}_{0}(t)\right) \boldsymbol{\Psi}_{0101}=2 \mathbf{B}_{0}(t)\left(\overline{\boldsymbol{\zeta}_{0}}, \overline{\boldsymbol{\zeta}_{1}}\right) \\
& a_{1} \boldsymbol{\zeta}_{1}+\left(\frac{d}{d t}-\mathbf{L}_{0}(t)\right) \boldsymbol{\Psi}_{0200}=\mathbf{B}_{0}(t)\left(\overline{\boldsymbol{\zeta}_{0}}, \overline{\boldsymbol{\zeta}_{0}}\right)
\end{aligned}
$$

where we define

$$
\mathbf{B}_{0}(t)=\mathbf{B}_{\Gamma_{c}, \mathcal{B}_{c}, \mathcal{R}_{c}}(t)
$$


Because of the $2 \pi$ - periodicity of $\mathbf{B}_{0}(t), \boldsymbol{\zeta}_{1}(t), \boldsymbol{\zeta}_{1}^{*}(t)$ and the fact that changing $t$ into $t+2 \pi$ changes the sign of $\boldsymbol{\zeta}_{0}$ and $\boldsymbol{\zeta}_{0}^{*}$, we observe immediately that half of the compatibility conditions are satisfied:

$$
\begin{aligned}
\left\langle\mathbf{B}_{0}(t)\left(\overline{\boldsymbol{\zeta}_{0}}, \overline{\boldsymbol{\zeta}_{1}}\right), \overline{\boldsymbol{\zeta}_{1}^{*}}\right\rangle=\left\langle\mathbf{B}_{0}(t)\left(\overline{\boldsymbol{\zeta}_{0}}, \overline{\boldsymbol{\zeta}_{1}}\right), \boldsymbol{\zeta}_{1}^{*}\right\rangle=0, \\
\left\langle\mathbf{B}_{0}(t)\left(\overline{\boldsymbol{\zeta}_{0}}, \overline{\boldsymbol{\zeta}_{0}}\right), \overline{\boldsymbol{\zeta}}_{0}^{*}\right\rangle=0,\left\langle\mathbf{B}_{0}(t)\left(\overline{\boldsymbol{\zeta}_{0}}, \overline{\boldsymbol{\zeta}_{0}}\right), \overline{\boldsymbol{\zeta}_{0}^{*}}\right\rangle=0 .
\end{aligned}
$$

The resonance conditions (17) and (19) lead to

$$
\left\langle\mathbf{B}_{0}(t)\left(\overline{\boldsymbol{\zeta}_{0}}, \overline{\boldsymbol{\zeta}_{1}}\right), \overline{\boldsymbol{\zeta}_{0}^{*}}\right\rangle=0,\left\langle\mathbf{B}_{0}(t)\left(\overline{\boldsymbol{\zeta}_{0}}, \overline{\boldsymbol{\zeta}_{0}}\right), \overline{\boldsymbol{\zeta}_{1}^{*}}\right\rangle=0
$$

and it remains the two last compatibility conditions leading to (here for $q$ odd, Hypothesis $2 \mathrm{a}$ and $n$ odd)

$$
a_{0}=2\left\langle\mathbf{B}_{0}(t)\left(\overline{\boldsymbol{\zeta}_{0}}, \overline{\boldsymbol{\zeta}_{1}}\right), \boldsymbol{\zeta}_{0}^{*}\right\rangle, \quad a_{1}=\left\langle\mathbf{B}_{0}(t)\left(\overline{\boldsymbol{\zeta}_{0}}, \overline{\boldsymbol{\zeta}_{0}}\right), \boldsymbol{\zeta}_{1}^{*}\right\rangle \text { in }(36)
$$

In the same way we find for $q$ odd

$$
\begin{aligned}
& a_{0}=2\left\langle\mathbf{B}_{0}(t)\left(\overline{\boldsymbol{\zeta}_{0}}, \boldsymbol{\zeta}_{1}\right), \boldsymbol{\zeta}_{0}^{*}\right\rangle, \quad a_{1}=\left\langle\mathbf{B}_{0}(t)\left(\boldsymbol{\zeta}_{0}, \boldsymbol{\zeta}_{0}\right), \boldsymbol{\zeta}_{1}^{*}\right\rangle \text { in }(37), \\
& a_{0}=2\left\langle\mathbf{B}_{0}(t)\left(\boldsymbol{\zeta}_{0},\left(\boldsymbol{\zeta}_{1}+\overline{\boldsymbol{\zeta}_{1}}\right)\right), \boldsymbol{\zeta}_{0}^{*}\right\rangle, \quad a_{1}=2\left\langle\mathbf{B}_{0}(t)\left(\boldsymbol{\zeta}_{0}, \overline{\boldsymbol{\zeta}_{0}}\right), \boldsymbol{\zeta}_{1}^{*}\right\rangle \text { in }(38) .
\end{aligned}
$$

Now for $q$ even, we find in the same way (see Appendix C)

$$
\begin{aligned}
& a_{0}=2\left\langle\mathbf{B}_{0}(t)\left(\boldsymbol{\zeta}_{0}, \mathbf{R}_{\pi / q} \boldsymbol{\zeta}_{1}\right), \boldsymbol{\zeta}_{0}^{*}\right\rangle, \quad a_{1}=\left\langle\mathbf{B}_{0}(t)\left(\mathbf{R}_{\pi / q} \boldsymbol{\zeta}_{0}, \mathbf{R}_{\pi / q} \boldsymbol{\zeta}_{0}\right), \boldsymbol{\zeta}_{1}^{*}\right\rangle \text { in }(C .2), \\
& a_{0}=2\left\langle\mathbf{B}_{0}(t)\left(\boldsymbol{\zeta}_{0}, \boldsymbol{\zeta}_{1}\right), \boldsymbol{\zeta}_{0}^{*}\right\rangle, \quad a_{1}=\left\langle\mathbf{B}_{0}(t)\left(\boldsymbol{\zeta}_{0}, \boldsymbol{\zeta}_{0}\right), \boldsymbol{\zeta}_{1}^{*}\right\rangle \text { in }(C .3), \\
& a_{0}=2\left\langle\mathbf{B}_{0}(t)\left(\boldsymbol{\zeta}_{0},\left(\boldsymbol{\zeta}_{1}+\mathbf{R}_{\pi / q} \boldsymbol{\zeta}_{1}\right)\right), \boldsymbol{\zeta}_{0}^{*}\right\rangle, \quad a_{1}=2\left\langle\mathbf{B}_{0}(t)\left(\boldsymbol{\zeta}_{0}, \mathbf{R}_{\pi / q} \boldsymbol{\zeta}_{0}\right), \boldsymbol{\zeta}_{1}^{*}\right\rangle \text { in (C.4). }
\end{aligned}
$$

An important remark for the computation of coefficients is that we can simplify things by noticing that we have sums of $q$ identical terms. In fact we have by construction

$$
\left\langle\boldsymbol{\zeta}_{j}, \boldsymbol{\zeta}_{j}^{*}\right\rangle=1, \quad j=0,1
$$

and since there are $q$ identical term in the scalar product with the $\boldsymbol{\zeta}_{j}$, this implies

$$
\begin{aligned}
& \frac{1}{4 \pi} \int_{0}^{4 \pi} \widehat{U}_{\mathbf{k}_{1}}(t) \cdot \widehat{U}_{\mathbf{k}_{1}}^{*}(t) d t=1 / q \\
& \frac{1}{2 \pi} \int_{0}^{2 \pi} \widehat{U}_{\mathbf{k}_{1}^{\prime}}(t) \cdot \widehat{U}_{\mathbf{k}_{1}^{\prime}}^{*}(t) d t=1 / q .
\end{aligned}
$$


Defining

$$
\begin{aligned}
& \boldsymbol{\xi}_{0}^{*}=q \widehat{U}_{\mathbf{k}_{1}}^{*}(t) e^{i \mathbf{k}_{1} \cdot \mathbf{x}}, \quad \boldsymbol{\xi}_{1}^{*}=q \widehat{U}_{\mathbf{k}_{1}^{\prime}}^{*}(t) e^{i \mathbf{k}_{1}^{\prime} \cdot \mathbf{x}} \\
& \boldsymbol{\xi}_{1}^{\prime}=\mathbf{R}_{\pi / 2 q} \boldsymbol{\xi}_{1}, \quad \boldsymbol{\xi}_{1}^{\prime *}=\mathbf{R}_{\pi / 2 q} \boldsymbol{\xi}_{1}^{*} \text { for Hyp } 2 \mathrm{~b}
\end{aligned}
$$

we then obtain

$$
\left\langle\boldsymbol{\xi}_{j}, \boldsymbol{\xi}_{i}^{*}\right\rangle=\delta_{i j}, \quad i, j=0,1 .
$$

We are then able to rewrite coefficients $a_{0}, a_{1}$ in (36), (37), (C.2) and (C.3):

$$
\begin{aligned}
a_{0} & =2\left\langle\mathbf{B}_{0}(t)\left(\mathbf{R}_{(2 n+q) \pi / q} \boldsymbol{\xi}_{0}, \mathbf{R}_{n \pi / q} \boldsymbol{\xi}_{1}\right), \boldsymbol{\xi}_{0}^{*}\right\rangle \\
& +2\left\langle\mathbf{B}_{0}(t)\left(\mathbf{R}_{(q-2 n) \pi / q} \boldsymbol{\xi}_{0}, \mathbf{R}_{-n \pi / q} \boldsymbol{\xi}_{1}\right), \boldsymbol{\xi}_{0}^{*}\right\rangle \\
a_{1} & =2\left\langle\mathbf{B}_{0}(t)\left(\mathbf{R}_{-n \pi / q} \boldsymbol{\xi}_{0}, \mathbf{R}_{n \pi / q} \boldsymbol{\xi}_{0}\right), \boldsymbol{\xi}_{1}^{*}\right\rangle
\end{aligned}
$$

and in (38) and (C.4):

$$
\begin{aligned}
a_{0} & =2\left\langle\mathbf{B}_{0}(t)\left(\mathbf{R}_{(2 n+q-1) \pi / q} \boldsymbol{\xi}_{0}, \mathbf{R}_{(n-1) \pi / q} \boldsymbol{\xi}_{1}^{\prime}\right), \boldsymbol{\xi}_{0}^{*}\right\rangle \\
& +2\left\langle\mathbf{B}_{0}(t)\left(\mathbf{R}_{(q+1-2 n) \pi / q} \boldsymbol{\xi}_{0}, \mathbf{R}_{-n \pi / q} \boldsymbol{\xi}_{1}^{\prime}\right), \boldsymbol{\xi}_{0}^{*}\right\rangle \\
a_{1} & =2\left\langle\mathbf{B}_{0}(t)\left(\mathbf{R}_{(1-n) \pi / q} \boldsymbol{\xi}_{0}, \mathbf{R}_{n \pi / q} \boldsymbol{\xi}_{0}\right), \boldsymbol{\xi}_{1}^{*}\right\rangle .
\end{aligned}
$$

Remark 11. If equations $\mathbf{k}_{j}+\mathbf{k}_{l}+\mathbf{k}_{s}=0$ or $\mathbf{k}_{j}^{\prime}+\mathbf{k}_{l}^{\prime}+\mathbf{k}_{s}^{\prime}=0$ have solutions, which is the case for $q=3 k, k \geq 2$, then there are new quadratic coefficients in such cases.

For a given set $(n, q)$, the values of the coefficients $a_{0}$ and $a_{1}$ can be found numerically. As described in Appendix B, we compute the critical values of the physical parameters $\left(\mathcal{R}_{c}, \mathcal{B}_{c}\right)$ such that the hypothesis 1 and 2 a are verified. The kernel of the linearized operator $\frac{d}{d t}-\mathbf{L}_{\Gamma_{c}, \mathcal{R}_{c}, \mathcal{B}_{c}}$ of section 3.1 are computed numerically via a shooting method. The change $t \rightarrow-t$ that transforms the previous operator into the adjoint one, permits to compute the the kernel of $\frac{d}{d t}+\mathbf{L}_{\Gamma_{c}, \mathcal{R}_{c}, \mathcal{B}_{c}}^{*}$ of section 3.2 without supplementary effort. In Fig. E.5, we present an example of a numerical kernel of eq. (29).

The numerical values of the coefficients $a_{0,1}$ defined by (E.3) are then directly obtained through a Gauss-Kronrod quadrature formula. For $n=$ $1, q=5$, we get $a_{0}=-0.194$ and $a_{1}=0.125$, whereas for $n=1, q=7$, we get $a_{0}=-0.217$ and $a_{1}=0.081$. In both cases $a_{0} a_{1}<0$ which allows to satisfy necessary stability conditions of bimodal solutions found at section 4.2.2, for suitable values of the parameter combinations $\mu_{0}, \mu_{1}$. 

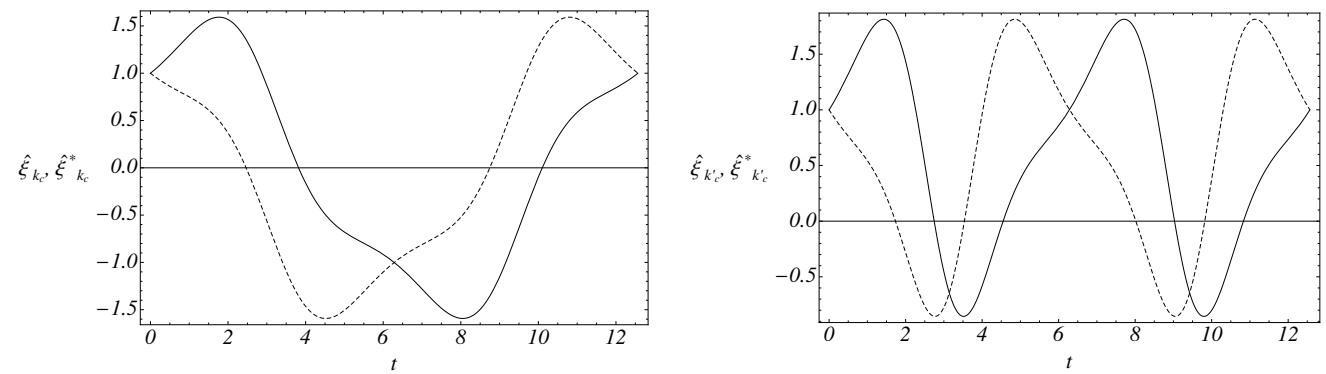

Figure E.5: component $u$ of $\boldsymbol{\xi}_{k}$ (see (30)), and $v$ (not normalized) of $\boldsymbol{\xi}_{k}^{*}$ (see (31)) in the kernels of the linearized operator (solid curve) and its adjoint (dashed curve). (left) $4 \pi$ periodic solutions. (right) $2 \pi$ periodic solution. These solutions have been computed in the critical case $n=1, q=5$, i.e. $\left(\mathcal{B}_{c}, \mathcal{R}_{c}\right)=(1.27,4.07)$.

\section{Acknowledgement}

G.I. thanks A. Rucklidge and M. Silber for fruitful discussions relative to this work.

[1] J. McLaughlin. Good Vibrations. American Scientist 4 (1998) 342-349.

[2] E. Chladni. Die Akustik, Leipzig Breitkopf \& Härtel, 1802.

[3] M. Faraday. On the forms and states of fluids on vibrating elastic surfaces, Phil. Trans. Roy. Soc. London 121 (1831) 299-318.

[4] L. Matthiessen. Akustische Versuche, die kleinsten Transversalwellen der Flüssigkeiten betreffend. Annln Phys., Lpz 134 (1868) 107-117.

[5] L. Matthiessen. Über die Transversalschwingungen tönender tropfbarer und elastischer Flüssigkeiten. Annln Phys. Lpz 141 (1870) 375-393.

[6] Lord Rayleigh. On the crispations of fluid resting upon a vibrating support. Philos. Mag. 16 (1883) 50-58.

[7] T. Benjamin, F. Ursell. The stability of the plane free surface of a liquid in vertical periodic motion. Proc. Roy. Soc. Lond. 1225 (1954) 505-515.

[8] K. Kumar, L. Tuckerman. Parametric instability of the interface between two fluids. J. Fluid. Mech 279 (1994) 49-68. 
[9] J. Bechhoeffer, V. Ego, S. Manneville, B. Johnson. An experimental study of the onset of parametrically pumped surface waves in viscous fluids. J. Fluid. Mech. 288 (1995) 325-350.

[10] O. Lioubashevski, J. Fineberg, L. Tuckerman. Scaling of the transition to parametrically driven surface waves in highly dissipative systems. Phys. Rev. E 55 (1997) R3832-3835.

[11] E. Cerda, E. Tirapegui. Faraday's instability for viscous fluids. Phys. Rev. Lett. 78 (1997) 859-863.

[12] G.Iooss, A.M.Rucklidge. On the existence of quasipattern solutions of the Swift-Hohenberg equation. J.Nonlinear Sci. 20, 3 (2010) 361-394.

[13] W. Edwards, S. Fauve. Patterns and quasipatterns in the Faraday experiment, J. Fluid. Mech. 278 (1994) 123-148.

[14] A. Ezerskii, M. Rabinovich, M. Reutov, I. Starobinets. Spatiotemporal chaos in the parametric excitation of a capillary ripple. J. Exp. Theor. Phys. 64 (1986) 1228-1236.

[15] N. Tufillaro, R. Ramshankar, J. Gollub. Order-Disorder Transition in Capillary Ripples. Phys. Rev. Lett. 62 (1989) 422-425.

[16] S. Ciliberto, S. Douady, S. Fauve. Investigating Space-Time Chaos in Faraday Instability by Mean of the Fluctuations of the Driving Acceleration. Europhys. Lett 15 (1991) 23-28.

[17] K. Kumar, K Bajaj. Competing patterns in the Faraday experiment. Phys. Rev. E 52 (1995), R4606-4609.

[18] A. Kudrolli, J. Gollub. Patterns and spatiotemporal chaos in parametrically forced surface waves: a systematic survey at large aspect ratio. Physica D 97 (1996) 133-154.

[19] D. Binks, W, van de Water. Nonlinear pattern formation of Faraday waves. Phys. Rev. Lett. 78 (1997) 4043-4046.

[20] N. Prinet, D. Juric, L.S. Tuckerman. Numerical Simulation of Faraday waves,.J. Fluid Mech. 635 (2009) 1-26. 
[21] W.S. Edward, S. Fauve. Parametrically excited quasicrystalline surface waves. Phys. Rev. E 47 (1993) R788.

[22] M-T. Westra, D. J. Binks, W. van de Water. Patterns of Faraday waves. J. Fluid. Mech. 496 (2003) 1-32.

[23] J. Miles. Nonlinear Faraday Resonance. J. Fluid. Mech. 146 (1984) 285302.

[24] S. Milner. Square patterns and secondary instabilities in driven capillary waves. J. Fluid. Mech. 225, (1991) 81-100.

[25] J. Miles. On Faraday Waves. J. Fluid. Mech. 248 (1993) 671-683.

[26] A.Rucklidge, M.Silber. Design of parametrically forced patterns and quasipatterns. SIAM J. Appl. Dyn. Syst. 8,1, (2009) 298-347.

[27] W. Zhang, J. Viñals. Square patterns and quasipatterns in weakly damped Faraday waves. Phys. Rev. E 53 (1996) R4283-4286.

[28] W. Zhang, J. Viñals. Pattern formation in weakly damped parametric surface waves. J. Fluid. Mech. 336 (1997) 301-330.

[29] W. Zhang, J. Viñals. Pattern formation in weakly damped parametric surface waves driven by two frequency components. J. Fluid. Mech. 341 (1997) 225-244.

[30] P. Chen and J. Viñals. Amplitude equation and pattern selection in Faraday waves. Phys. Rev. E 60 (1999) 559-570.

[31] N. Rojas, M. Argentina, E. Cerda, E. Tirapegui. "Inertial lubrication theory" . Phys. Rev. lett. 104 (2010) , 187801-187804.

[32] N.O. Rojas, M. Argentina, E. A. Cerda, E. L. Tirapegui. Faraday patterns in lubricated thin films, Eur. Phys. J. D 62 (2011) 25-31.

[33] A.M.Rucklidge, W.J.Rucklidge. Convergence properties of the 8, 10 and 12 mode representations of quasipatterns. PhysicaD 178 (1-2) (2003) $62-82$. 